\title{
Stable ergodicity for smooth compact Lie group extensions of hyperbolic basic sets
}

\author{
MICHAEL FIELD $\dagger$, IAN MELBOURNE $\ddagger$ and ANDREI TÖRÖK $\dagger \S$ \\ $\dagger$ Department of Mathematics, University of Houston, Houston, TX 77204-3008, USA \\ (e-mail:mf@uh.edu,torok@math.uh.edu) \\ $\ddagger$ Department of Mathematics and Statistics, University of Surrey, Guildford, \\ Surrey GU2 7XH, UK \\ (e-mail: ism@math.uh.edu) \\ $\S$ Institute of Mathematics of the Romanian Academy, PO Box 1-764, \\ RO-70700, Bucharest, Romania
}

(Received 30 September 2003 and accepted in revised form 18 March 2004)

\begin{abstract}
We obtain sharp results for the genericity and stability of transitivity, ergodicity and mixing for compact connected Lie group extensions over a hyperbolic basic set of a $C^{2}$ diffeomorphism. In contrast to previous work, our results hold for general hyperbolic basic sets and are valid in the $C^{r}$-topology for all $r>0$ (here $r$ need not be an integer and $C^{1}$ is replaced by Lipschitz). Moreover, when $r \geq 2$, we show that there is a $C^{2}$-open and $C^{r}$-dense subset of $C^{r}$-extensions that are ergodic. We obtain similar results on stable transitivity for (non-compact) $\mathbb{R}^{m}$-extensions, thereby generalizing a result of Niţică and Pollicott, and on stable mixing for suspension flows.
\end{abstract}

\section{Introduction}

In this paper we consider the stable transitivity and ergodicity of Lie group extensions over a hyperbolic basic set with a fixed base diffeomorphism. We also obtain stable transitivity and ergodicity results for suspension flows (fixed base map and varying roof function). Elsewhere [11], we show how our methods can be developed to prove, for example, that mixing hyperbolic flows are open and dense within the class of smooth non-trivial hyperbolic flows.

After a survey of some of the existing results on extensions by compact Lie groups, we describe in $\S 1.2$ our new results on compact group and $\mathbb{R}^{m}$-extensions over a hyperbolic basic set. In $\S 1.3$, we describe our results on suspension flows. In $\S 1.4$, we describe related results in [11]. 
1.1. Compact group extensions of a fixed hyperbolic basic set. In this subsection, we give a brief historical review of some of the previous work in this area that relates to our work.

Let $G$ be a compact connected Lie group. In 1975, Brin [9] proved the genericity (in the $C^{2}$-topology) of stable ergodicity for compact Lie group extensions of Anosov diffeomorphisms. Specifically, Brin showed that there was a $C^{1}$-open and $C^{1}$-dense set of transitive compact Lie group extensions of an Anosov diffeomorphism [9, Theorem 2.2] and deduced (using [10, Theorem 5.3]) that every $C^{2}$-transitive extension was Kolmogorov and, a fortiori, ergodic. More recently, using somewhat different methods, Adler et al [1] re-proved a variant of Brin's result that applied to circle extensions of Anosov diffeomorphisms of a torus. Specifically, they showed that there is a $C^{0}$-open and $C^{\infty}$-dense set of extensions of such diffeomorphisms that are ergodic. (The same result holds for toral extensions of a hyperbolic attractor [14].) Following the result of Adler et al [1], Parry and Pollicott [23] studied stability of mixing for toral extensions over aperiodic subshifts of finite type and over connected hyperbolic basic sets subject to a cohomological restriction.

Field and Parry [14] generalized these results to allow for extensions by compact connected non-Abelian Lie groups $G$. In the case that $G$ is semisimple, they proved that for all $\alpha \in(0,1)$ and $\infty \geq r \geq \alpha$, there is a $C^{\alpha}$-open, $C^{r}$-dense set of ergodic extensions over a general hyperbolic basic set. In the case that the basic set is an attractor, they obtained the same stability result for general compact connected Lie groups $G$. Extensions and variations of the results in $[14,23]$ may be found in $[12,13]$.

Unfortunately, the situation regarding toral extensions over general hyperbolic basic sets as reported in these works is less than satisfactory. Thus, in [23], the stability and density result for extensions of a subshift of finite type is proved in the $C^{\alpha}$-topology, $\alpha \in(0,1)$, but the open and dense set of ergodic $C^{\alpha}$-extensions contains no $C^{\beta}$-extensions, $\beta>\alpha$. A second issue is that the methods of $[\mathbf{1 4}, \mathbf{2 3}]$ require for toral extensions either that the base is a subshift of finite type or that the base is connected and a cohomological condition holds (the only examples known satisfying the cohomological condition are attractors/repellors).

In the present paper, we resolve these problems and give sharp results for compact Lie group extensions of general hyperbolic basic sets. Although our emphasis here is on skew (product) extensions, all of our results apply to principal $G$-extensions (see $[14, \S 5.3]$ ). Our results also apply to classes of partially hyperbolic $G$-invariant basic sets. For these applications, we refer to [12].

1.2. Results on group extensions. We suppose throughout that $\Phi$ is a fixed $C^{2}$-diffeomorphism of the compact manifold $M$, and that $\Lambda$ is a hyperbolic basic set for $\Phi$.

Definition 1.1. We say that $\Lambda$ is a hyperbolic basic set for the diffeomorphism $\Phi$ if $\Lambda$ is a locally maximal hyperbolic set, and $\Phi \mid \Lambda$ is topologically transitive. In addition, we require that the basic set $\Lambda$ is non-trivial, meaning that it does not coincide with a periodic orbit.

Definition 1.2. For $r>0$, we give the usual meaning to ' $C^{r}$ ' and the ' $C{ }^{r}$-topology' except that $C^{1}$-maps will be defined as Lipschitz. 
By $C^{r}(M, G)$ we denote the space of $C^{r}$-cocycles on $M$ with the $C^{r}$-topology.

THEOREM 1.3. For $r>0$, there exists a $C^{r}$-open and-dense subset $\mathcal{W}_{r}$ of $C^{r}(M, G)$ such that for all $f \in \mathcal{W}_{r}$, the skew extension $\Phi_{f}: \Lambda \times G \rightarrow \Lambda \times G$ is transitive. If $r \geq 2$, $\mathcal{W}_{r}$ is open in the $C^{2}$-topology on $C^{r}(M, G)$ and $C^{\infty}$-cocycles are $C^{[r]}$-dense in $\mathcal{W}_{r}$. An analogous result holds for principal $G$-extensions over $\Lambda$.

Remark 1.4. Theorem 1.3 is of main interest when $G$ is not semisimple or $\Lambda$ is not an attractor- $C^{\alpha}$-stability, $C^{\infty}$-density holds if $G$ is semisimple or $\Lambda$ is an attractor [14] for any $\alpha>0$. On the other hand, if $G=\mathbb{K}^{m}, r<1$ and $\Lambda$ is a subshift of finite type, then $\mathcal{W}_{r} \cap \mathcal{W}_{t}=\emptyset, 0<r<t$, and we conjecture that this may occur for values of $r \in[1,2)$.

In our study of ergodic properties of extensions, we suppose that $\mu$ is the unique equilibrium state associated to a Hölder continuous potential on $\Lambda$ [6]. It follows that $\Phi: \Lambda \rightarrow \Lambda$ is $\mu$-ergodic. We take Haar measure $h$ on $G$ and define the product measure $v=\mu \times h$ on $\Lambda \times G$. All skew extensions $\Phi_{f}$ are $v$-measure-preserving.

THEOREM 1.5. With the notation of Theorem 1.3, if $f \in \mathcal{W}_{r}$, then $\Phi_{f}$ is v-ergodic. An analogous result holds for principal $G$-extensions over $\Lambda$. If $\Phi: \Lambda \rightarrow \Lambda$ is mixing and $f \in \mathcal{W}_{r}$, then $\Phi_{f}$ is mixing.

Remark 1.6. The equivalence of ergodicity and transitivity for the situation described in Theorems 1.3 and 1.5 is a direct consequence of Livšic regularity (see §6). The last statement of Theorem 1.5 is a consequence of [14, Proposition 3.2.1]-if $\Phi$ is mixing, then stable ergodicity of $\Phi_{f}$ is equivalent to stable mixing of $\Phi_{f}$.

Before stating our next result, we recall some definitions from [19]. We continue to assume that $r>0$. Let $f \in C^{r}\left(M, \mathbb{R}^{m}\right)$ and define the corresponding skew extension $\Phi_{f}: \Lambda \times \mathbb{R}^{m} \rightarrow \Lambda \times \mathbb{R}^{m}$. Write $f_{n}=\sum_{j=0}^{n-1} f \circ \Phi^{j}$. Let $\mathcal{L}_{f}=\left\{f_{n}(x) \mid \Phi^{n} x=x\right\}$. We say that $f$ satisfies the separating condition if $\mathcal{L}_{f}$ is not contained on one side of a hyperplane through 0 in $\mathbb{R}^{m}$. Let $\mathcal{S}^{r} \subset C^{r}\left(M, \mathbb{R}^{m}\right)$ denote the set of cocycles that satisfy the separating condition. We remark that $\mathcal{S}^{r}$ is a non-empty open (but never dense) subset of $C^{r}\left(M, \mathbb{R}^{m}\right)$. Niţică and Pollicott [19] studied the case when $\Lambda=M$ is Anosov and proved that $\Phi_{f}$ is transitive for $f \in \mathcal{S}^{r}$.

THEOREM 1.7. For $r>0$, there exists a $C^{r}$-open and-dense subset $\mathcal{W}_{r}$ of $\mathcal{S}^{r}$ such that for all $f \in \mathcal{W}_{r}$, the skew extension $\Phi_{f}: \Lambda \times \mathbb{R}^{m} \rightarrow \Lambda \times \mathbb{R}^{m}$ is transitive. If $r \geq 2, \mathcal{W}_{r}$ is open in the $C^{2}$-topology on $C^{r}\left(M, \mathbb{R}^{m}\right)$ and $C^{\infty}$-cocycles are $C^{[r]}$-dense in $\mathcal{W}_{r}$.

Remark 1.8. If $\Lambda$ is a hyperbolic attractor, then we obtain the improved result that $\mathcal{W}_{r}$ is $C^{0}$-open in $\mathcal{S}^{r}$. Again, $C^{\alpha}$-openness, $\alpha \in[0,1)$, fails for general hyperbolic basic sets.

1.3. Suspension flows. We now describe our main result on the stability of weak mixing for suspensions flows over hyperbolic basic sets. Let $\mathcal{R}^{r}$ denote the space of strictly positive functions (roof functions) in $C^{r}(M, \mathbb{R})$. Each roof function $f \in \mathcal{R}^{r}$ defines a suspension flow $\Phi_{t}^{f}: \Lambda^{f} \rightarrow \Lambda^{f}$ on the suspension $\Lambda^{f}$. When $\Lambda=M$ is Anosov, it follows from the Anosov alternative [2] that $\Lambda^{f}$ is mixing for all non-constant $f \in \mathcal{R}^{r}$. 
THEOREM 1.9. For $r>0$, there exists a $C^{r}$-open and-dense subset $\mathcal{W}_{r}$ of $\mathcal{R}^{r}$ such that for all $f \in \mathcal{W}_{r}$, the suspension flow $\Phi_{t}^{f}: \Lambda^{f} \rightarrow \Lambda^{f}$ is weak mixing. If $r \geq 2, \mathcal{W}_{r}$ is open in the $C^{2}$-topology on $C^{r}(M, \mathbb{R})$ and $C^{\infty}$ roof functions are $C^{[r]}$-dense in $\mathcal{W}_{r}$.

Remark 1.10. If $\Lambda$ is a hyperbolic attractor, then we obtain the improved result that $\mathcal{W}_{r}$ is $C^{0}$-open in $\mathcal{R}^{r}$. Once again, $C^{\alpha}$-openness, $\alpha \in[0,1)$, fails for general hyperbolic basic sets.

Remarks 1.11. (1) On a suspension $\Lambda^{f} \cong \Lambda \times \mathbb{R} / \sim$ we consider the equilibrium state $\mu^{f}=\mu \times \ell / \int_{\Lambda} f d \mu$ where $\ell$ is Lebesgue measure and $\mu$ is an equilibrium state associated to a Hölder potential on $\Lambda$. However, weak mixing of $\Phi_{t}^{f}$ is independent of the choice of $\mu$ and is equivalent to topological weak mixing (see for example [21, Proposition 6.2]). Hence, we suppress any reference to the measure in the results concerning mixing of hyperbolic (suspension) flows.

(2) Moreover, it follows from standard results (see, for example, [24, p. 418] and [7, Remark 3.5] for statements and references) that weak-mixing suspension flows are automatically strong-mixing, Kolmogorov and even Bernoulli (so for each $t \in \mathbb{R} \backslash\{0\}$ the diffeomorphism $\Phi_{t}^{f}: \Lambda^{f} \rightarrow \Lambda^{f}$ is isomorphic to a Bernoulli shift). Consequently, it follows from Theorem 1.9 that an open and dense set of suspension flows over a hyperbolic basic set are Bernoulli.

1.4. Varying the base diffeomorphism. Let $n=\operatorname{dim} M$ and restrict to $C^{s}$-diffeomorphisms, $\Phi \in \operatorname{Diff}^{s}(M), s \geq 2 n+1$. If we allow the base diffeomorphism $\Phi$ to vary in the context of Theorems 1.3 and 1.5, then it can be shown using the methods of [11] that compact group extensions $\Phi_{f}: \Lambda_{\Phi} \times G \rightarrow \Lambda_{\Phi} \times G$ are transitive/ergodic for $(\Phi, f)$ lying in a $C^{2 n+1} \times C^{2}$-open and $C^{s} \times C^{r}$-dense subset of $\operatorname{Diff}^{s}(M) \times C^{r}(M, G)$. Similar results in the context of Theorems 1.7 and 1.9 hold for $\mathbb{R}^{m}$-extensions and suspension flows.

1.5. Brief remarks on proofs and extensions. Brin's original proof of stable transitivity of compact Lie group extensions over an Anosov diffeomorphism uses transitivity properties of the stable and unstable foliations, and the well-known 'quadrilateral construction'. In a related paper [8], Brin gives a direct construction of maximal transitivity components which uses the structure of the strong stable and unstable foliations of the extension. While the existence of maximal transitivity components holds under the assumption that the base is a basic set (see [22, §5]), the quadrilateral argument appears to require at least some local path connectivity in the invariant foliations of the basic set. Thus, the quadrilateral argument works for general hyperbolic attractors (see [12]), but fails for subshifts of finite type. A common theme of more recent work is the use of a result of Keynes and Newton [16] together with the Livšic regularity theorem [17], [20, Theorem 3.1]. This approach allows one to prove that the ergodic components naturally define a partition by closed sets and leads to simple arguments for stable ergodicity when $G$ is semisimple (see [14]). Matters are not so simple when $G$ is Abelian, and the proof of Parry and Pollicott's result on toral extensions over subshifts of finite type makes use of the Ruelle transfer operator. Since we are interested in $C^{r}$ results, $r \geq 1$ 
(and cannot assume that maps are expanding), we avoid this approach here. Our proof of Theorem 1.3 for subshifts of finite type depends on obtaining good asymptotic bounds on the average of $\mathbb{R}$-valued cocycles along a sequence of periodic points asymptotic to a homoclinic point. Using our methods, we give in $\$ 3$ a simple proof of the original abstract result of Parry and Pollicott, and in $\$ 5$ a more geometric and very explicit proof using results of Williams on geometric realization of subshifts of finite type [26]. For $C^{r}$-extensions, we consider subshifts of finite type in $\S 4$. We deduce the result for general basic sets using the fact that basic sets contain (many) subshifts of finite type together with a routine argument based on either Keynes and Newton and Livšic regularity or the existence of maximal transitivity components.

Finally, we note that the results in this paper go far beyond the context of hyperbolic dynamics. For example, the results on group extensions over a hyperbolic basic set $\Lambda$ generalize immediately to the case where $\Lambda$ is a locally maximal topologically transitive set such that: (i) $\Lambda$ contains a transverse homoclinic point, and (ii) $\Lambda$ supports results on Livšic regularity whereby measurable solutions to certain cohomological equations have continuous versions. See Remark 6.4.

Remark 1.12. The heart of this paper, $\S 4$, is concerned with proving Theorem 1.5 in the special case when $X$ is a subshift of finite type and $G$ is the circle group. The remainder of the results are then consequences of this special case. See $\S 6$ for the proof of Theorem 1.5 for general $\Lambda$ and $G, \S 7$ for the proof of Theorem 1.7, and $\S 8$ for the proof of Theorem 1.9 .

\section{Preliminaries}

Throughout this paper we assume that $M$ is a compact smooth Riemannian manifold without boundary.

2.1. Transverse homoclinic points and Smale's theorem. Let $\Phi: M \rightarrow M$ be a diffeomorphism of $M$. Suppose that $P \in M$ is a hyperbolic periodic point for $\Phi$ of prime period $q$. Suppose that $x_{H}$ is a transverse homoclinic point for the periodic orbit through $P$. Replacing $\Phi$ by $\Phi^{q}$, it will be no loss of generality (see below) to assume that $x_{H}$ is a transverse homoclinic point for the hyperbolic fixed point $P$ of $\Phi$.

We say that a subset $X$ of $M$ is an $H$-set for $\Phi$ if:

(1) $X$ is a compact locally maximal $\Phi$-invariant subset of $M$;

(2) $\Phi \mid X$ is transitive;

(3) $\Phi \mid X$ is hyperbolic;

(4) $X$ is conjugate to a subshift of finite type.

We recall Smale's theorem (see [15, Theorem 6.5.5, Exercise 6.5.1]).

THEOREM 2.1. Let $\Phi: U \rightarrow M$ be an embedding of the open set $U \subset M$, and $P, x_{H} \in U$. Assume that $x_{H}$ is a transverse homoclinic point for the hyperbolic fixed point $P$ of $\Phi$. Then there is an $H$-set for $\Phi$ containing $P$ and $x_{H}$. 
2.2. Function spaces. Suppose that $X$ is an $H$-set for $\Phi$. We choose a (closed) cover of $X$ by mutually disjoint contractible neighborhoods, with smooth boundaries. Let $U$ denote the closed neighborhood of $X$ with smooth boundary that is defined by the cover. Without loss of generality, we may and shall regard $U$ as a subset of $\mathbb{R}^{n}, n=\operatorname{dim}(M)$.

For $r>0$, let $C=C^{r}(U, \mathbb{R})$ denote the space of $C^{r} \mathbb{R}$-valued cocycles on $U$. If $r$ is an integer, $C^{r}$ has the usual meaning except if $r=1$ when we regard $C^{1}(U, \mathbb{R})$ as the space of Lipschitz continuous functions on $U$. With this proviso, we take the usual $C^{r}$ topology on $C$. We let \|\|$_{r}$ denote a choice of $C^{r}$-norm on $C$. With respect to this norm, $C$ is a Banach space.

Definition 2.2. An element $g \in C$ is called an $X$-coboundary if we can choose a continuous function $h: X \rightarrow \mathbb{R}$ such that

$$
g=h \circ \Phi-h .
$$

We denote the subspace of $C$ consisting of $X$-coboundaries by $B^{r}=B^{r}(U, \mathbb{R})$ or just $B$ if the meaning is clear from the context.

Remarks 2.3. (1) We do not ask that an $X$-coboundary be a coboundary on $U$. Nor do we require that the function $h$ occurring in the definition is more than continuous on $X$. In fact, it follows from Livšic regularity that it suffices to assume $h \mid X$ is measurable relative to an equilibrium state on $X$, as it then follows we can choose $h \mid X$ to be $C^{\alpha}$ (Hölder) continuous if $g$ is $C^{\alpha}, \alpha \in(0,1]$.

(2) In the sequel, we drop the prefix ' $X$ ' and just refer to coboundaries.

PROPOSITION 2.4. For $r>0$, the space $B^{r}(U, \mathbb{R})$ is a $C^{0}$-closed subspace of $C^{r}(U, \mathbb{R})$.

Proof. It follows from the Livšic theorems that an element $f \in C^{r}(U, \mathbb{R})$ is a coboundary if and only if the average of $f$ over each periodic orbit of $\Phi$ in $X$ is zero.

Definition 2.5. We let $L(U, \mathbb{R})$ denote the subspace of $C$ consisting of functions which are locally constant on $X$. Let $V=V^{r}(U, \mathbb{R})$ denote the $C^{r}$-closure of $L(U, \mathbb{R})$.

Henceforth, if $r>0$ is given, we always take the corresponding $C^{r}$-topologies on the subspaces $B$ and $V$.

Remarks 2.6. (1) Let $r>0$ and $C^{r}(X)$ denote the space of $\mathbb{R}$-valued functions $f: X \rightarrow \mathbb{R}$ which admit a $C^{r}$-extension $E(f) \in C^{r}(U, \mathbb{R})$. We similarly let $V^{r}(X)$ denote the subspace of $C^{r}(X)$ consisting of functions which admit a $C^{r}$-extension $E(f) \in V^{r}(U, \mathbb{R})$. If $r \in(0,1)$ and $\infty \geq s>r$, then $V^{r}(X) \supset C^{s}(X)$. That is, in the $C^{r}$-topology on $C^{r}(X)$, every $C^{s}$-function, $s>r$, can be $C^{r}$-approximated by a locally constant function. This result is (obviously) not true if $r \geq 1$.

(2) Every locally constant function $f$ on $X$ has an extension $E(f) \in V^{\infty}(U, \mathbb{R})$.

(3) If $r$ is not an integer, then $C^{s}, \infty \geq s>r$ functions are not dense in $C^{r}(U, \mathbb{R})$ (or $C^{r}(X)$ ). Density follows, by the Stone-Weierstrass approximation theorem, if $r$ is an integer. 
2.3. Criteria for the transitivity and ergodicity of toral extensions. Let $\mathbb{K}^{m}$ denote the $m$-dimensional torus, regarded as a compact Abelian group. We start by recalling a special case of a general result of Keynes and Newton giving necessary and sufficient conditions for the ergodicity of a compact group extension.

THEOREM 2.7. [16, §2] Let $\Phi: X \rightarrow X$ be an ergodic transformation of the probability space $(X, \mu)$. Let $f: X \rightarrow \mathbb{K}^{m}$ be $\mu$-measurable. Then $\Phi_{f}: X \times \mathbb{K}^{m} \rightarrow X \times \mathbb{K}^{m}$ is $\mu \times h$-ergodic if and only if for all $\ell=\left(\ell_{1}, \ldots, \ell_{m}\right) \in \mathbb{Z}^{m}, \ell \neq 0$, the equation

$$
w \circ \Phi(x)=w(x) \prod_{i=1}^{m} f_{i}^{\ell_{i}}(x), \quad \text { almost every } x,
$$

has no measurable solutions $w: X \rightarrow \mathbb{K}$.

Let $f: M \rightarrow \mathbb{K}^{m}$ be a $C^{r}$-cocycle and $\Phi_{f}: M \times \mathbb{K}^{m} \rightarrow M \times \mathbb{K}^{m}$ denote the corresponding $\mathbb{K}^{m}$-extension defined by $\Phi_{f}(x, g)=(\Phi(x), g f(x))$. Restricting to the $H$-set $X \subset M$, we obtain a $\mathbb{K}^{m}$-extension $\Phi_{f}: X \times \mathbb{K}^{m} \rightarrow X \times \mathbb{K}^{m}$.

For the remainder of the section, and following Parry and Pollicott [23], we give criteria for the ergodicity and transitivity of the extension $\Phi_{f}$. We do this in terms of a fixed equilibrium state $\mu$ on $X$. However, the resulting conditions are independent of the equilibrium state and depend only on the cocycle $f$. We refer the reader to [23] for details we omit.

Let $f \in C^{r}\left(U, \mathbb{K}^{m}\right), f=\left(f_{1}, \ldots, f_{m}\right)$. Using our cover of $U$ by contractible sets, we may choose $F_{i} \in C^{r}(U, \mathbb{R}), 1 \leq i \leq m$, such that $f_{i}=\exp \left(2 \pi \imath F_{i}\right)$ (on $U$ ). Note that we may suppose that the $F_{i}$ depend continuously on $f$ in the $C^{r}$-topology. Let $S(F)$ denote the $\mathbb{R}$-vector subspace of $C^{r}(U, \mathbb{R})$ spanned by $F_{1}, \ldots, F_{m}$.

LEMMA 2.8. (cf. [14]) The extension $\Phi_{f}$ is ergodic if $S(F) \cap(B+L)=\{0\}$. If $m=1$, then $\Phi_{f}$ is stably ergodic if and only if $F \notin \overline{B+L}$ (closure in $C^{r}$ ).

Proof. It follows from Theorem 2.7 and Livšic regularity that if $\Phi_{f}$ is not ergodic then there exists $\left(\ell_{1}, \ldots, \ell_{m}\right) \neq(0, \ldots, 0)$, such that

$$
g \circ \Phi=f_{1}^{\ell_{1}} \cdots f_{m}^{\ell_{m}} g
$$

has a non-trivial solution $g \in C^{0}(X, \mathbb{K})$. Noting that $g \circ \Phi / g \in C^{r}(U, \mathbb{R})$ and our definition of $B^{r}(U, \mathbb{R})$, it follows easily that we may choose $M, H \in C^{r}(U, \mathbb{R})$ such that

$$
M+H=\sum_{i=1}^{m} \ell_{i} F_{i}
$$

where

(1) $\quad M$ is a locally constant integer-valued function on $X$;

(2) $H \in B^{r}(U, \mathbb{R})$.

If equation (2.3) has solutions, then $S(F) \cap(B+L) \neq\{0\}$. That is, if $S(F) \cap(B+L)=\{0\}$ then $\Phi_{f}$ is ergodic.

Suppose $m=1$. If $F \in \overline{B+L}$, then we can find $F^{\prime}$ arbitrarily $C^{r}$-close to $F$ such that $F^{\prime}=b+v$ where $b$ is a coboundary and $v$ is locally constant on $X$ and takes only rational values on $X$. It follows that for some integer $\ell, \ell F^{\prime}=\tilde{b}+m$, where $m$ is locally constant 
and integer-valued on $X$ and $\tilde{b}$ is a coboundary. Setting $f^{\prime}=\exp \left(2 \pi \iota F^{\prime}\right)$, it follows that $\Phi_{f^{\prime}}$ is not ergodic. Hence, $\Phi_{f}$ cannot be stably ergodic.

Remarks 2.9. It follows from Lemma 2.8 that in order to prove the $C^{r}$-genericity of stable ergodicity (or transitivity) for $\mathbb{K}^{m}$-extensions, it suffices to prove that $\overline{B+L}$ is of codimension at least $m$. In the case of $C^{\alpha}$-extensions, $r=\alpha \in(0,1)$, Parry and Pollicott prove that $\overline{B+L}$ is of infinite codimension. They prove further that (a) $B+V$ is closed (recall that $V=\bar{L})$, (b) $C /(B+V)$ is not separable. We are interested in the case $r>1$, especially the case when $r$ is an integer. When $r$ is integral, $C / \overline{B+L}$ is separable. We do not know, at this time, whether $B+V$ is closed.

(2) We concentrate throughout on proving stable ergodicity. In the case that $\Phi$ is topologically mixing, then stable ergodicity is enough to guarantee stable mixing $[14, \S 3.2]$.

2.4. Reduction to the case of a fixed point and $\mathbb{K}$-extensions. Continuing with our previous notations, suppose that $X$ is an $H$-set for the diffeomorphism $\Phi$. As usual, we set $\Phi=\Phi \mid X$ and let $r \geq 2$. In order to prove the $C^{r}$-genericity of stably ergodic $C^{r} \mathbb{K}$-extensions, it suffices, by Lemma 2.8, to find (a single) $F \in C^{r}(U, \mathbb{R})$ such that $F \notin \overline{B+L}$. In fact, we shall prove rather more.

THEOREM 2.10. Let $r>0$. Suppose that $\Phi$ has a fixed point $p$ and $x_{H}$ is a transverse homoclinic point for $p$. Let $D^{\star} \subset U$ be a neighborhood of the closure of the $\Phi$-orbit of $x_{H}$ and $D \subset D^{\star}$ be a neighborhood of $p$. We can choose $F \in C^{r}(U, \mathbb{R})$ and $a$ $C^{r}$-neighborhood $\mathcal{W}$ of $F$ in $C^{r}(U, \mathbb{R})$ such that:

(1) $\operatorname{supp}(F) \subset D$;

(2) if $F^{\prime} \in \mathcal{W}$, then $F^{\prime} \notin \overline{B+L}$;

(3) if $H \in C^{r}(U, \mathbb{R})$ is such that $\operatorname{supp}(H) \cap D^{\star}=\emptyset$, then $F^{\prime}+H \notin \overline{B+L}$, all $F^{\prime} \in \mathcal{W}$;

(4) if $r \geq 2$, then we may take $\mathcal{W}$ to be open in the $C^{2}$-topology on $C^{r}(U, \mathbb{R})$;

(5) if $r \geq 2$ is an integer, then $C^{\infty}$ cocycles are dense in $\mathcal{W}$.

Next, we make a simple extension of Theorem 2.10 to allow for periodic points.

LEMMA 2.11. Let $r>0$. Suppose that $\Phi$ has a periodic point $p \in X$ of prime period $q$ and $x_{H}$ is a transverse homoclinic point for $p$. Let $D^{\star} \subset U$ be a neighborhood of the closure of the $\Phi$-orbit of $x_{H}$ and $D \subset D^{\star}$ be a neighborhood of $p$. We can choose $F \in C^{r}(U, \mathbb{R})$, and a $C^{r}$-neighborhood $\mathcal{W}$ of $F$ in $C^{r}(U, \mathbb{R})$ such that the conclusions of Theorem 2.10 are valid.

Proof. Shrink $D$ so that the subsets $\Phi^{-i}(D)$ are disjoint for $i=0,1, \ldots, q-1$. Since $\Phi^{q}(p)=p$, it is immediate from Theorem 2.10 that we can choose $F \in C^{r}(U, \mathbb{R})$ supported in $D$ with the required properties with $\Phi$ replaced by $\Phi^{q}$. In particular, $F \notin \overline{B_{q}+L}$ where $B_{q}$ is the space of $C^{r}$-coboundaries for $\Phi^{q}$. This proves stable ergodicity of $\left(\Phi^{q}\right)_{f}$ where $f=e^{2 \pi \imath F}$.

Define $\tilde{F}=\sum_{i=0}^{q-1} F \circ \Phi^{i}$. Then $\tilde{F}$ coincides with $F$ on $D^{\star}$ and so by Theorem 2.10(3) $\tilde{F} \notin \overline{B_{q}+L}$. Again, $\left(\Phi^{q}\right)_{\tilde{f}}$ is stably ergodic, where $\tilde{f}=e^{2 \pi \imath \tilde{F}}$. But $\left(\Phi^{q}\right)_{\tilde{f}}=\left(\Phi_{f}\right)^{q}$, 
so we have shown stable ergodicity of $\left(\Phi_{f}\right)^{q}$ and hence $\Phi_{f}$. In particular, it follows from Lemma 2.8 that $F \notin \overline{B+L}$.

There remains the case of $\mathbb{K}^{m}$-extensions, $m>1$. Choose a set of $m$ disjoint periodic orbits. By the preceding arguments, we may choose $F_{1}, \ldots, F_{m} \notin \overline{B+L}$ such that the supports of the $F_{i}$ are mutually disjoint. Obviously, $F_{1}, \ldots, F_{m}$ are linearly independent $\bmod \overline{B+L}$. In particular, $\operatorname{codim}_{C}(\overline{B+L})=\infty$.

\section{Outline of the proof and the theorem of Parry and Pollicott}

We continue to assume that $\Phi: X \rightarrow X$ is an $H$-set for $\Phi$. Let $r>0$. Suppose that $p \in X$ is a hyperbolic fixed point for $\Phi$.

3.1. Outline of proof. Suppose $F \in C^{r}(U, \mathbb{R})$. Let $x \in X$. If $x$ is periodic of prime period $n$, we define the average of $F$ along the $\Phi$-orbit of $x$ by

$$
\operatorname{Av}(F, x)=\sum_{i=0}^{n-1} F\left(\Phi^{i}(x)\right) .
$$

If $x$ is a homoclinic point to a periodic point $p$ and $\operatorname{Av}(F, p)=0$, then we define

$$
\operatorname{Av}(F, x)=\sum_{i=-\infty}^{\infty} F\left(\Phi^{i}(x)\right)
$$

Using our assumption that $F$ is $C^{r}$, and certainly Hölder, it is easy to show that this sum converges.

Suppose that $x_{H} \in X$ is a transverse homoclinic point for $p$. Let $\left(p_{N}\right) \subset X$ be a sequence of periodic points such that $p_{N} \rightarrow x_{H}$. It follows from the continuity of $\Phi$ that for all $i \in \mathbb{Z}, \Phi^{i}\left(p_{N}\right) \rightarrow \Phi^{i}\left(x_{H}\right)$, as $N \rightarrow \infty$.

Suppose that $F$ is $C^{r}$-close to $B+L$, so that $F=b+\ell+g, b \in B, \ell \in L,\|g\|_{r}$ small. Without loss of generality, we may suppose that $\operatorname{Av}(F, p)=\operatorname{Av}(\ell, p)=0$. In particular,

$$
A_{N}(F)=\operatorname{Av}\left(F, x_{H}\right)-\operatorname{Av}\left(F, p_{N}\right)
$$

is well defined for all $N \geq 1$.

It is well known, and simple to verify, that $\operatorname{Av}\left(b, p_{N}\right)=\operatorname{Av}\left(b, x_{H}\right)=0$ for all $N \geq 1$ and that $\operatorname{Av}\left(\ell, p_{N}\right)=\operatorname{Av}\left(\ell, x_{H}\right)$ for $N$ sufficiently large. Hence, for $N$ large, we have

$$
A_{N}(F)=A_{N}(g) \text {. }
$$

It is now an easy matter to construct explicit cocycles $F \notin B+L-$ we require only that $A_{N}(F)$ be not eventually zero. It is also possible to construct cocycles $F \notin \overline{B+L}$ by comparing the asymptotics on each side of (3.4). Specifically, we obtain (i) upper bounds for the right-hand side of (3.4) for all small $g$, and (ii) lower bounds for the left-hand side of (3.4) for an explicitly constructed cocycle $F$. In this way, we show that $F-g \notin B+L$ provided that $\|g\|_{r}$ is sufficiently small. In fact, since our construction only depends on averages near the $\Phi$-orbit of $x_{H}$, it will follow that $g$ can take arbitrary values outside of some arbitrarily small neighborhood of the closure of the $\Phi$-orbit of $x_{H}$. 
In order to obtain our estimates, we will need to estimate the location of the periodic points in the sequence $\left(p_{N}\right)$ rather precisely. We remark that the details of our proof are much easier if we make the assumption that $\Phi$ is smoothly linearizable at $p$. However, this assumption is not necessary for the proofs of Theorems 1.3, 1.5, 1.7 and 1.9 which do not require any non-resonance conditions on the linearization of $\Phi$ at the fixed point.

We note that the construction involving $x_{H}$ and $p_{N}$ described above has been used independently by Bonatti et al [3].

3.2. The theorem of Parry and Pollicott. We conclude this section with an illustration of how our methods give a proof of the stability result of Parry and Pollicott for (abstract) subshifts of finite type that avoids any consideration of the Ruelle transfer operator and, as we shall see, generalizes to smooth cocycles.

Let $X$ be a subshift of finite type. Given $\theta \in(0,1)$ we define the metric $d(x, y)=\theta^{n}$ where $n$ is the largest non-negative integer such that $x_{i}=y_{i}$ for $|i|<n$. Let $F_{\theta}(X)$ be the space consisting of all functions $f: X \rightarrow \mathbb{R}$ that are Lipschitz with respect to this metric. Let $|f|_{\infty}$ denote the sup-norm and $|f|_{\theta}$ the least Lipschitz constant. Then, $F_{\theta}(X)$ is a Banach space under the norm $\|f\|_{\theta}=\max \left\{|f|_{\infty},|f|_{\theta}\right\}$.

Let $B=B_{\theta}(X) \subset F_{\theta}(X)$ denote the subspace of coboundaries and $L \subset F_{\theta}(X)$ be the subspace of locally constant functions. If $U$ is an open subset of $X$, we let $L_{\theta}(U)$ denote the subspace of $F_{\theta}(X)$ consisting of functions which are locally constant on $U$.

Remark 3.1. If $b=c \circ \sigma-c \in B_{\theta}$ then by Livšic regularity the cobounding function $c: X \rightarrow \mathbb{R}$ can be chosen to lie in $F_{\theta}$. In particular, $c$ is continuous.

We give an elementary proof of the following result of Parry and Pollicott.

THEOREM 3.2. [23] The subspace $\overline{B_{\theta}+L}$ has infinite codimension in $F_{\theta}$.

We start by proving a special case of part of Theorem 3.2.

PROPOSITION 3.3. Suppose that the shift map $\sigma: X \rightarrow X$ has a fixed point $p$ and that $x_{H}$ is a homoclinic point for $p$. If $D^{\star}$ is any open neighborhood of the closure of the $\sigma$-orbit of $x_{H}$, then we can choose $\tilde{f} \in F_{\theta}(X)$, such that:

(1) $\tilde{f} \notin \overline{B_{\theta}+L}$;

(2) $\operatorname{supp}(\tilde{f}) \subset D^{\star}$;

(3) if $g \in F_{\theta}(X)$ has $\operatorname{supp}(g) \cap D^{\star}=\emptyset$, then $\tilde{f}+g \notin \overline{B_{\theta}+L}$;

(4) for some $c>0,\|\tilde{f}-(b+\ell)\|_{\theta} \geq c$, all $b \in B_{\theta}$ and $\ell \in L_{\theta}\left(D^{\star}\right)$.

In particular, $\overline{B_{\theta}+L} \neq F_{\theta}(X)$.

We present the proof of Proposition 3.3 in the special case where $X$ is the full shift $\{0,1\}^{\mathbb{Z}}$ on two symbols and leave the details of the general case to the reader (note that the general case can be reduced to this case by recoding).

We denote points $x \in\{0,1\}^{\mathbb{Z}}$ by $\cdots x_{-1} \cdot x_{0} x_{1} \cdots$. Let $p=0^{\infty} \cdot 0^{\infty}$ and suppose for simplicity that the homoclinic point is $x_{H}=0^{\infty} \cdot 10^{\infty}$. We let $\left(p_{N}\right)$ be the sequence of periodic points of period $2 N+1$ defined by $p_{N}=\left[0^{N} \cdot 10^{N}\right]$. Note that $p_{N} \rightarrow x_{H}, N \rightarrow \infty$. 
Given $F \in F_{\theta}(X)$ satisfying $F(p)=0$, define $A_{N}(F)=\operatorname{Av}\left(F, x_{H}\right)-\operatorname{Av}\left(F, p_{N}\right)$. We have

$$
\begin{aligned}
A_{N}(F) & =\sum_{j=-\infty}^{\infty} F\left(\sigma^{j} x_{H}\right)-\sum_{j=-N}^{N} F\left(\sigma^{j} p_{N}\right) \\
& =\sum_{j=-N}^{N}\left[F\left(\sigma^{j} x_{H}\right)-F\left(\sigma^{j} p_{N}\right)\right]+\sum_{|j|>N} F\left(\sigma^{j} x_{H}\right)
\end{aligned}
$$

\section{LEMMA 3.4.}

(a) If $b \in B_{\theta}$, then $b(p)=0$ and $A_{N}(b)=0$ for all $N$.

(b) If $\ell$ is locally constant and $\ell(p)=0$, then there exists $N_{0}$ such that $A_{N}(\ell)=0$ for all $N \geq N_{0}$.

(c) If $g \in F_{\theta}$ and $g(p)=0$, then $\left|A_{N}(g)\right| \leq 4|g|{ }_{\theta} \theta^{N} /(1-\theta)$ for all $N$.

Proof. Parts (a) and (b) are well known and elementary. We prove part (c). Since $p_{N}$ and $x_{H}$ first differ in the $\pm(2 N+1)$ th position, $\left|g\left(\sigma^{j} p_{N}\right)-g\left(\sigma^{j} x_{H}\right)\right| \leq|g|_{\theta} \theta^{2 N+1-|j|}$ for $|j| \leq 2 N+1$. Hence,

$$
\left|\sum_{j=-N}^{N}\left[g\left(\sigma^{j} p_{N}\right)-g\left(\sigma^{j} x_{H}\right)\right]\right| \leq|g|_{\theta} \theta^{N+1}\left(\theta^{N}+2 \theta^{N-1}+\cdots+2\right) \leq \frac{2|g|{ }_{\theta} \theta^{N+1}}{1-\theta} .
$$

Similarly, since $g(p)=0$, we can write

$$
\sum_{|j|>N} g\left(\sigma^{j} x_{H}\right)=\sum_{|j|>N}\left[g\left(\sigma^{j} x_{H}\right)-g(p)\right]
$$

which is dominated by $2|g|_{\theta}\left(\theta^{N}+\theta^{N+1}+\cdots\right) \leq 2|g|_{\theta} \theta^{N} /(1-\theta)$.

LEMMA 3.5. Let $C_{q}$ be the cylinder of length $2 q+1$ given by $C_{q}=\{x \in X \mid x=$ $\left.\cdots 0^{q} \cdot 00^{q} \cdots\right\}$. Define

$$
f=\sum_{q \geq 1} \theta^{q}\left(1-I_{C_{q}}\right)
$$

Then $f \in F_{\theta}, f(p)=0$ and $A_{N}(f)=2\|f\|_{\theta} \theta^{N} /(1-\theta)$.

Proof. It is immediate from the definitions that $|f|_{\infty} \leq \theta /(1-\theta)$ and $|f|_{\theta} \leq \theta /(1-\theta)$. Also, $x_{H} \notin C_{q}$ for all $q$ so that $f\left(x_{H}\right)=\theta /(1-\theta)$. Hence, $f \in F_{\theta}$ with $\|f\|_{\theta}=\theta /(1-\theta)$. Since $p \in C_{q}$ for all $q, f(p)=0$.

Next, we note that $\sigma^{j} x_{H} \in C_{|j|-1}-C_{|j|}$ for each $j$. Hence $f\left(\sigma^{j} x_{H}\right)=\theta^{|j|} /(1-\theta)$.

The same calculation for $p_{N}$ shows that $\sigma^{j} p_{N} \in C_{|j|-1}-C_{|j|}$ and hence $f\left(\sigma^{j} p_{N}\right)=$ $\theta^{|j|} /(1-\theta)$ for $|j| \leq N$. We conclude that

$$
A_{N}(f)=\sum_{|j|>N} f\left(\sigma^{j} x_{H}\right)=\frac{2 \theta^{N+1}}{\left(1-\theta^{2}\right)}
$$

Proof of Proposition 3.3. Let $f$ be the function defined in Lemma 3.5. Suppose for contradiction that $f \in \overline{B_{\theta}+L}$. Then, for any $\varepsilon>0$, there exists $b \in B_{\theta}, \ell \in L$ and $g \in F_{\theta}$ with $\|g\|_{\theta}<\varepsilon$, such that $f=b+\ell+g$. Replacing $\ell$ by $\ell+g(p)$ and $g$ by $g-g(p)$ 
we can assume without loss of generality that $\ell(p)=g(p)=0$ while maintaining $|g|_{\theta}<\varepsilon$. Since $A_{N}$ is additive, we have $A_{N}(f)=A_{N}(b)+A_{N}(\ell)+A_{N}(g)$. By Lemma 3.4, there exists $N_{0}$ such that $A_{N}(f)=A_{N}(g)$ for all $N \geq N_{0}$. For such $N$, we have

$$
2\|f\|_{\theta} \theta^{N} /(1-\theta) \leq 4 \varepsilon \theta^{N} /(1-\theta),
$$

and hence $\|f\|_{\theta} \leq 2 \varepsilon$. Since $\varepsilon>0$ is arbitrary, we obtain the desired contradiction, proving the first statement of Proposition 3.3. Since the argument only depended on averages of functions along the orbits of $x_{H}$ and $p_{N}$, for arbitrarily large $N$, it is clear that if $g \in F_{\theta}$ is supported outside of some open neighborhood $D^{\star}$ of the closure of the orbit of $x_{H}$, then $f+g \notin \overline{B_{\theta}+L}$.

If $b \in B_{\theta}$, and $\ell \in L_{\theta}\left(D^{\star}\right)$, then $A_{N}(f-(b+\ell))=A_{N}(f)-A_{N}(\ell)=A_{N}(f)$, for sufficiently large $N$. By Lemma 3.4, $\left|A_{N}(f-(b+\ell))\right| \leq 4|f-(b+\ell)|{ }_{\theta} \theta^{N} /(1-\theta)$ for all $N$. Hence,

$$
|f-(b+\ell)|_{\theta} \geq \frac{1}{4} \theta^{-N}(1-\theta) A_{N}(f)
$$

for sufficiently large values of $N$. But, by Lemma 3.5, $A_{N}(f)=2\|f\|_{\theta} \theta^{N} /(1-\theta)$. Hence it follows that $\|f-(b+\ell)\|_{\theta} \geq|f-(b+\ell)|_{\theta} \geq\|f\|_{\theta} / 2$, for all $b, \ell$.

Finally, to complete the proof, note that it is no loss of generality to assume that $D^{\star}$ is open and closed. It follows that if we define $\tilde{f}=f \chi_{D^{\star}}$, then $\tilde{f} \in F_{\theta}(X)$ and $\|\tilde{f}\|_{\theta} \geq\|f\|_{\theta}$. Equation (3.5) continues to hold, with $\tilde{f}$ replacing $f$. Since $A_{N}(\tilde{f})=$ $A_{N}(f)$ for sufficiently large $N$, we deduce that $\|\tilde{f}-(b+\ell)\|_{\theta} \geq\|f\|_{\theta} / 2=\theta /(2(1-\theta))$ for all $b, \ell$.

Following the argument in the general case (Lemma 2.11), we can allow for periodic points.

Lemma 3.6. Suppose that $p \in X$ is a periodic orbit and that $x_{H}$ is a homoclinic point for the orbit of $p$. If $D^{\star}$ is any open neighborhood of the closure of the $\sigma$-orbit of $x_{H}$, then we can choose $\tilde{f} \in F_{\theta}$, such that:

(1) $\tilde{f} \notin \overline{B_{\theta}+L}$;

(2) $\operatorname{supp}(\tilde{f}) \subset D^{\star}$;

(3) if $g \in F_{\theta}$ has $\operatorname{supp}(g) \cap D^{\star}=\emptyset$, then $\tilde{f}+g \notin \overline{B_{\theta}+L}$;

(4) for some $c>0,\|\tilde{f}-(b+\ell)\|_{\theta} \geq c$, all $b \in B_{\theta}$ and $\ell \in L_{\theta}\left(D^{\star}\right)$.

The theorem of Parry and Pollicott is an immediate consequence of the following result.

THEOREM 3.7. There is a sequence of disjoint open and closed sets $U_{j}$ and functions $f_{j} \in F_{\theta}(X)$ with the following properties:

(i) $\operatorname{supp}\left(f_{j}\right) \subset U_{j}$;

(ii) $\left\|f_{j}-(b+\ell)\right\|_{\theta} \geq 1$ for all $b \in B_{\theta}(X)$ and $\ell \in L_{\theta}\left(U_{j}\right)$.

Proof. Let $x^{j}$ be a sequence of periodic points lying on distinct periodic orbits $O\left(x^{j}\right)$. Choose $x_{H}^{j}$ homoclinic to $x^{j}$ and let $O\left(x_{H}^{j}\right)$ denote the homoclinic orbit through $x_{H}^{j}$. The points $x^{j}$ and $x_{H}^{j}$ can be chosen so that there are disjoint open and closed sets $U_{j}$ such that $O\left(x^{j}\right) \cup O\left(x_{H}^{j}\right) \subset U_{j}$. 
It follows from Lemma 3.6 that for each $j$, we can find a cocycle $f_{j}$, and constant $c_{j}>0$, such that $\operatorname{supp}\left(f_{j}\right) \subset U_{j}$ and $\left\|f_{j}-(b+\ell)\right\|_{\theta} \geq c_{j}$, for all $b \in B, \ell \in L_{\theta}\left(U_{j}\right)$. Replacing $f_{j}$ by $f_{j} / c_{j}$, it follows that we can require $\left\|f_{j}-(b+\ell)\right\|_{\theta} \geq 1$, for all $b \in B$, $\ell \in L_{\theta}\left(U_{j}\right)$.

\section{Proof of Theorem 2.10}

As outlined in $\S 3.1$, we will construct a sequence of periodic points $\left(\mathbf{p}_{N}\right)$ approaching the homoclinic point $x_{H}$. In Proposition 4.8 we give general estimates for the position of $\mathbf{p}_{N}$ under the assumption that $\Phi$ is linear on the stable and unstable manifolds of the fixed point $p$. We can and do improve these estimates on $\mathbf{p}_{N}$ if we assume that $\Phi$ is $C^{2}$-linearizable at $p($ Remark 4.10(3)).

In the remainder of the section, we consider the problems of obtaining upper bound estimates on $A_{N}(F)$ and finding functions $F$ for which the growth of $A_{N}(F)$ matches the upper bound. So as to simplify the exposition, we make some linearizability assumptions that we remove in $\S 4.9$. Specifically, in $\S \S 4.2$ and 4.4-4.8 we assume that $\Phi$ is linear on the stable and unstable manifolds of the fixed point $p$. We also assume in our verification of the upper bounds for $A_{N}(F), F$ at least $C^{2}$, that $\Phi$ is $C^{2}$-linearizable at $p$ (Lemma 4.13). Indeed, if $\Phi$ is not $C^{2}$-linearizable at $p$, then we have to allow for slower decay of $A_{N}(F)$ (see §4.9). Finally, we remark that results and methods are a little different when $F$ is $C^{r}$, $r \in(0,2)$.

4.1. The local model. We always assume that $\Phi$ is $C^{2}$. It follows that there will be $C^{2}$-local stable and unstable manifolds through the fixed point $p$. We use these invariant manifolds as the basis for a local $C^{2}$-coordinate system at $p$. Thus, we regard $p$ as the origin of the vector space $\mathbb{R}^{m}=\mathbb{E}^{s} \oplus \mathbb{E}^{u}$ with the local stable (respectively, unstable) manifold through $p$ contained in $\mathbb{E}^{s}$ (respectively, $\mathbb{E}^{u}$ ). We choose coordinates on $\mathbb{E}^{s}, \mathbb{E}^{u}$ so that $D \Phi(0)=G$ is in real Jordan normal form, with Jordan blocks $J_{1}, \ldots, J_{k}$.

We denote the set of generalized eigenspaces of $G$ by $\mathcal{E}$. Abusing notation, we will typically label elements of $\mathcal{E}$ by the associated eigenvalue. Thus, we label eigenvalues $\mu_{i}, \lambda_{j} \in \mathcal{E}$ so that

$$
\left|\mu_{1}\right| \leq\left|\mu_{2}\right| \leq \cdots \leq\left|\mu_{S}\right|<1<\left|\lambda_{T}\right| \leq \cdots \leq\left|\lambda_{1}\right| .
$$

In particular, $S+T=k$ and each $\rho \in \mathcal{E}$ will be associated to the Jordan block $J_{\rho}$. We let $p_{\rho}$ denote the algebraic multiplicity of $\rho$ (thus, for real $\rho, J_{\rho}$ will be a $p_{\rho} \times p_{\rho}$ square matrix, whereas for $\rho$ complex, $J_{\rho}, J_{\bar{\rho}}$ act on the same generalized eigenspace of real dimension $2 p_{\rho}$ ). We have the decompositions

$$
\mathbb{E}^{s}=\bigoplus_{i=1}^{S} \mathbb{E}_{\mu_{i}}, \quad \mathbb{E}^{u}=\bigoplus_{j=1}^{T} \mathbb{E}_{\lambda_{j}},
$$

where $\mathbb{E}_{\rho}$ is the generalized eigenspace associated to $\rho \in \mathcal{E}$. We define $G_{s}=G \mid \mathbb{E}^{s}$, $G_{u}=G \mid \mathbb{E}^{u}$.

We denote coordinates on $\mathbb{E}^{s}$ by $\mathbf{x}=\left(\mathbf{x}_{1}, \ldots, \mathbf{x}_{S}\right)$, where each $\mathbf{x}_{i}=\left(x_{i 1}, \ldots, x_{i p_{\mu_{i}}}\right) \in$ $\mathbb{E}_{\mu_{i}} \cong \mathbb{K}^{p_{\mu_{i}}}$, where $\mathbb{K}=\mathbb{R}, \mu_{i}$ real, and $\mathbb{K}=\mathbb{R}^{2} \cong \mathbb{C}, \mu_{i}$ complex. We similarly denote coordinates on $\mathbb{E}^{u}$ by $\mathbf{y}=\left(\mathbf{y}_{1}, \ldots, \mathbf{y}_{T}\right)$ and follow the same labeling conventions 


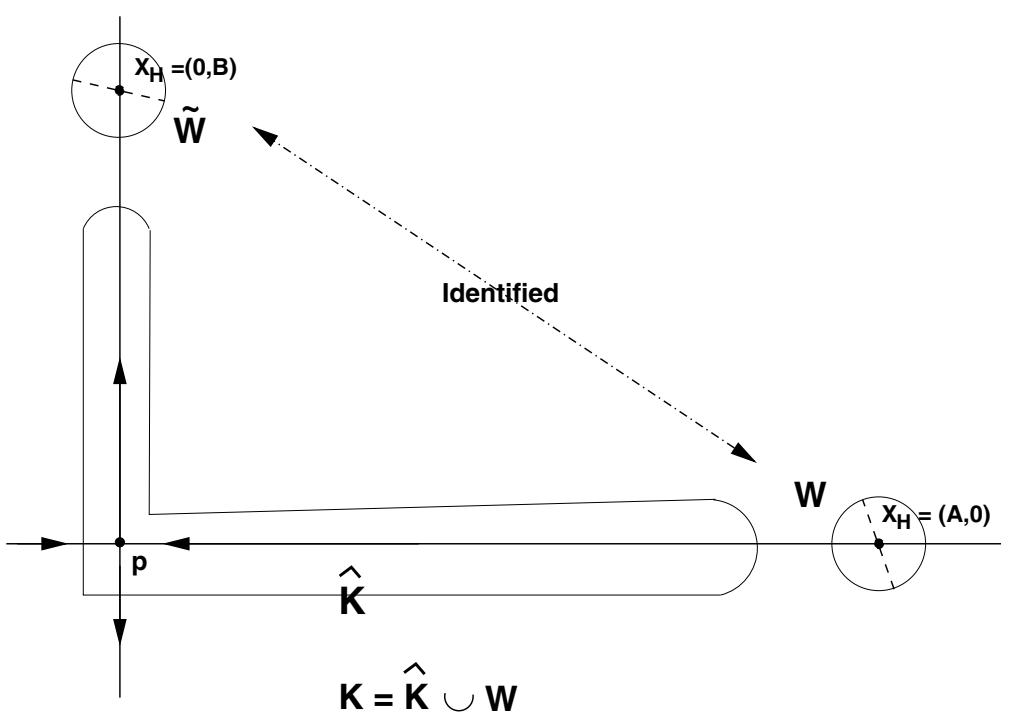

FIGURE 1. Basic local setup near the $\Phi$-orbit of $x_{H}$.

as for the stable subspaces. For our proofs, we usually assume eigenvalues are real-the assumption of complex eigenvalues presents no new difficulties but does complicate the notation a little.

Let $x_{H} \in \mathbb{E}^{s}$ be a transverse homoclinic point for $p$. Let $\tilde{x}_{H} \in \mathbb{E}^{u}$ be the point corresponding to $x_{H}$, now regarded as lying on the unstable manifold of $p$-see Figure 1 . Let $O\left(x_{H}\right)$ denote the $\Phi$-orbit of $x_{H}$. Note that the forward orbit of $x_{H}$ is contained in $\mathbb{E}^{s}$, while the backward orbit of $x_{H}=\tilde{x}_{H}$ is contained in $\mathbb{E}^{u}$ and that we regard $x_{H}$ and $\tilde{x}_{H}$ as identified. Let $O\left(x_{H}\right)^{\star}=O\left(x_{H}\right) \backslash\left\{x_{H}\right\} \subset \mathbb{E}^{s} \cup \mathbb{E}^{u}$ (no identification). Let $W, \tilde{W}$ be neighborhoods of $x_{H}, \tilde{x}_{H}$ chosen so that $(W \cup \tilde{W}) \cap O\left(x_{H}\right)=\left\{x_{H}\right\}$. We regard $W, \tilde{W}$ as identified (that is, in the ambient manifold.) Let $K$ be an open neighborhood of $\overline{O\left(x_{H}\right)}$ which is the union of $W \cup \tilde{W}$ together with an open neighborhood $\hat{K}$ of $\overline{O\left(x_{H}\right)^{\star}}$ disjoint from $W \cup \tilde{W}$. We may suppose that $K, W, \tilde{W}$ are chosen so that $\Phi(W) \subset \hat{K}$, $\Phi^{-1}(\tilde{W}) \subset \hat{K}$.

From now on, we regard $\Phi$ as defined on $K$ with the understanding that if $z \in K$ then $\Phi^{n}(z)$ is defined provided that the iterates of $z$ up to and including $\Phi^{n}(z)$ all lie in $K$. Henceforth, all our computations, perturbations and estimates will be done inside $K$. Of course, everything translates back to the ambient manifold $M$.

4.2. Basic expansion and contraction rates. For the present we assume that $\Phi$ is linear when restricted to neighborhoods of $p$ in $\mathbb{E}^{s}$ and $\mathbb{E}^{u}$. This is a mild restrictionif $\Phi$ is sufficiently smooth only finitely many non-resonance conditions are required on eigenvalues of $D \Phi(0)\left|\mathbb{E}^{s}, D \Phi(0)\right| \mathbb{E}^{u}$ for linearizability. In particular, we do not make the stronger requirement that $\Phi$ is $C^{2}$-linearizable at $p$. We indicate later (in $\S 4.9$ ) how we can remove the assumption of linearizability on the invariant manifolds at the cost of incurring some minor extra technical detail. However, including the detail at this point would only serve to needlessly complicate our exposition. 
Write

$$
x_{H}=\mathbf{A}=\left(\mathbf{A}_{1}, \ldots, \mathbf{A}_{S}\right) \in \bigoplus_{i=1}^{S} \mathbb{E}_{\mu_{i}}, \quad \tilde{x}_{H}=\mathbf{B}=\left(\mathbf{B}_{1}, \ldots, \mathbf{B}_{T}\right) \in \bigoplus_{j=1}^{T} \mathbb{E}_{\lambda_{j}} .
$$

LemMA 4.1. Let $J$ be a $p \times p$ Jordan block over $\mathbb{C}$ with eigenvalue $\rho \neq 0$. Let $\left[a_{i j}^{n}\right]$ denote the matrix of $J^{n}, n \in \mathbb{Z}$. Then,

$$
\begin{aligned}
a_{i j}^{n} & =0, \quad i>j, \\
& =\left(\begin{array}{c}
n \\
j-i
\end{array}\right) \rho^{n+i-j}, \quad i \leq j .
\end{aligned}
$$

In particular, given $p$, there exist $C, c>0$, independent of $n, i, j$, such that for $|n| \geq j-i$,

$$
c|n|^{j-i}|\rho|^{n+i-j} \leq\left|a_{i j}^{n}\right| \leq C|n|^{j-i}|\rho|^{n+i-j}, \quad n \in \mathbb{Z}, 1 \leq i \leq j \leq p .
$$

Proof. The proof is elementary and omitted.

For $m \geq 0$, we define

$$
\begin{aligned}
& \mathbf{A}^{m}=G_{s}^{m}(\mathbf{A}), \\
& \mathbf{B}^{m}=G_{u}^{-m}(\mathbf{B}) .
\end{aligned}
$$

We have $\lim _{m \rightarrow \infty} \mathbf{A}^{m}, \mathbf{B}^{m}=0$.

Fix $i \in\{1, \ldots, S\}$. If $\mathbf{A}_{i}=0$, then $\mathbf{A}_{i}^{m}=0$, for all $m \geq 0$. If $\mathbf{A}_{i} \neq 0$, write $\mathbf{A}_{i}^{m}=\left(A_{i 1}^{m}, \ldots, A_{i p}^{m}\right) \neq 0, m \geq 0$. We may find $p_{i} \in\{2, \ldots, p\}$, such that $A_{i p_{i}-1}^{m} \neq 0$, $m \geq 0, A_{i j}^{m}=0, j \geq p_{i}$, all $m \geq 0$. We similarly define positive integers $q_{j}$ for the $\mathbf{B}_{j}^{m}$ components.

Lemma 4.2. Let $\mathbf{A}_{i} \neq 0$ and define $p_{i} \in\{2, \ldots, p\}$ as above. There exist $m=m(i) \geq 0$, $C, c>0$, such that for all $n \geq m$ :

(1) $A_{i 1}^{n} \neq 0$;

(2) $\quad C\left|A_{i j}^{n}\right| \geq\left(\begin{array}{c}n \\ p_{i}-1-j\end{array}\right)\left|A_{i p_{i}-1}^{0}\right|\left|\mu_{i}\right|^{n} \geq c\left|A_{i j}^{n}\right|, j \in\left\{1, \ldots, p_{i}-1\right\}$.

We have a similar result for the non-zero $\mathbf{B}_{j}$ terms.

Proof. A straightforward application of Lemma 4.1.

Remark 4.3. A consequence of Lemma 4.2 is that for large values of $n, A_{i 1}^{n}$ is the dominant term in $\mathbf{A}_{i}^{n}$. Indeed, it follows from Lemma 4.2 that we can choose $c>0$ so that for $n \geq m$, $\left|A_{i j}^{n}\right| /\left|A_{i 1}^{n}\right| \leq c n^{1-j}$.

Let $\mathcal{E}_{\star}^{s}$ denote the set of eigenvalues $\mu_{i}$ for which $\mathbf{A}_{i} \neq 0$. We similarly define $\mathcal{E}_{\star}^{u}$.

Let $\tilde{\mu}$ be the largest value of $\left|\mu_{i}\right|, \mu_{i} \in \mathcal{E}_{\star}^{s}$ and $\tilde{\lambda}^{-1}$ be the largest value of $\left|\lambda_{j}^{-1}\right|$, $\lambda_{j} \in \mathcal{E}_{\star}^{u}$. We define

$$
\Psi=\max \left(\tilde{\mu}, \tilde{\lambda}^{-1}\right) .
$$

We say there is a $\Psi$-resonance if we can find $i, j$ such that $\tilde{\mu}=\left|\mu_{i}\right|, \tilde{\lambda}^{-1}=\left|\lambda_{j}^{-1}\right|$ and $\mu_{i} \lambda_{j}=1$. 
Let $\mathcal{C}^{s} \subset\{1, \ldots, S\}$ consist of those $i$ for which $\mu_{i} \in \mathcal{E}_{\star}^{s}$ and $\left|\mu_{i}\right|=\tilde{\mu}$. Let $p$ denote the maximum of $p_{i}, i \in \mathcal{C}^{s}$. Let $\bar{l}$ denote a value of $i$ for which $p_{i}=p$ and set $\bar{a}=p-1$. Thus, $A_{\bar{i} \bar{a}}^{m} \neq 0, m \geq m(\bar{l})$, and, if $\left|\mu_{i}\right|=\tilde{\mu}$, then $A_{i a}^{m}=0, a>\bar{a}$.

Let $\mathcal{C}^{u} \subset\{1, \ldots, T\}$ consist of those $j$ for which $\mu_{j} \in \mathcal{E}_{\star}^{u}$ and $\left|\lambda_{j}\right|=\tilde{\lambda}$. Let $q$ denote the maximum of $q_{j}$ over $j \in \mathcal{C}^{u}$. Just as above, we let $\bar{j}, \bar{b}$ denote the corresponding values of $j$ and $q-1$. In particular, $B_{\bar{j} \bar{b}}^{n} \neq 0, n \geq n(\bar{\jmath})$.

Let $\alpha=\min \{-\ln |\tilde{\lambda}|, \ln |\tilde{\mu}|\} / \ln \Psi$. If $\Psi=|\tilde{\mu}|$, define $m(p, q)=((p-1) \alpha+q-1) /$ $(1+\alpha)$, otherwise set $m(p, q)=(p-1+(q-1) \alpha) /(1+\alpha)$. For $N \in \mathbb{N}$ and $0<r<2$, define

$$
\begin{aligned}
\beta_{N} & = \begin{cases}N^{p-1} \Psi^{N} & \text { if } \Psi=\tilde{\mu}>\tilde{\lambda}^{-1}, \\
N^{q-1} \Psi^{N} & \text { if } \Psi=\tilde{\lambda}^{-1}>\tilde{\mu}, \\
N^{\max (p, q)-1} \Psi^{N} & \text { if } \Psi=\tilde{\mu}=\tilde{\lambda}^{-1},\end{cases} \\
\bar{\beta}_{N} & =N^{m(p, q)}\left(\Psi^{N}\right)^{\alpha /(1+\alpha)}, \\
\bar{\beta}_{N}(r) & = \begin{cases}\left(\bar{\beta}_{N}\right)^{r}, & \text { if } \alpha(r-1)<1, \\
N\left(\bar{\beta}_{N}\right)^{r}=N^{m(p, q)(1+\alpha) / \alpha+1} \Psi^{N} & \text { if } \alpha(r-1)=1, \\
\beta_{N} & \text { if } \alpha(r-1)>1,\end{cases} \\
\gamma_{N} & = \begin{cases}\beta_{N} & \text { if not } \Psi \text {-resonant, } \\
N^{p+q-1} \Psi^{N} & \text { if } \Psi \text {-resonant. }\end{cases}
\end{aligned}
$$

Remark 4.4. In the simplest case, where $G$ is semisimple and there is no $\Psi$-resonance, we have $\gamma_{N}=\beta_{N}=\Psi^{N}$. If $G$ is semisimple and there is a $\Psi$-resonance, we have $\beta_{N}=\Psi^{N}$ and $\gamma_{N}=N \Psi^{N}$. We only need the terms $\bar{\beta}_{N}$ and $\bar{\beta}_{N}(r)$ when considering cocycles of class $C^{r}, r<2$. Note that $\gamma_{N}=\bar{\beta}_{N}(2)$.

4.3. Technical lemmas. In this section we state, usually without proof, some useful elementary lemmas.

Lemma 4.5. Given $p \geq 0$, there exists $C>0$, independent of $n$, such that

$$
n^{p} \beta_{n}^{2} \leq C \Psi^{n / 2} \beta_{n} .
$$

Lemma 4.6. Let $p, q, \bar{m}, \bar{m}^{\star}, N \in \mathbb{N}$. For $x \neq 0$, define

$$
\begin{aligned}
\chi_{N}(x) & =\max \left(\left(N-\bar{m}-m^{\star}\right)^{p}|x|^{N-m^{\star}},\left(N-\bar{m}-m^{\star}\right)^{q}|x|^{\bar{m}}\right), \quad x \neq 1, \\
& =\left(N-\bar{m}-m^{\star}\right)^{p+q+1}, \quad x=1 .
\end{aligned}
$$

There exist constants $c=c(x, p, q), C=C(x, p, q)>0$, independent of $N, \bar{m}, m^{\star}$, such that given $\bar{m}, \bar{m}^{\star}$, we can choose $N^{\star} \in \mathbb{N}$ such that for $N \geq N^{\star}$,

$$
C \chi_{N}(x) \geq\left|\sum_{n=\bar{m}}^{N-m^{\star}}\left(\begin{array}{c}
n-\bar{m} \\
p
\end{array}\right)\left(\begin{array}{c}
-N+n+m^{\star} \\
q
\end{array}\right) x^{n}\right| \geq c \chi_{N}(x),
$$

with the proviso that if $x=-1, p=q=0$, then $N-m^{\star}-\bar{m}$ is even. 
Proof. Suppose that $x \neq 1$. It is straightforward to verify that

$$
\begin{aligned}
& \sum_{n=\bar{m}}^{N-m^{\star}}\left(\begin{array}{c}
n-\bar{m} \\
p
\end{array}\right)\left(\begin{array}{c}
-N+n+m^{\star} \\
q
\end{array}\right) x^{n} \\
& \quad=\frac{x^{\bar{m}+p}}{p ! q !}\left(\frac{d}{d x}\right)^{p}\left[x^{N-\bar{m}-m^{\star}+q}\left(\frac{d}{d x}\right)^{q}\left[\frac{x^{-N+\bar{m}+m^{\star}}-x}{1-x}\right]\right] .
\end{aligned}
$$

The result in the case $x \neq \pm 1$ or $x=-1, p+q \neq 0$ follows easily from this identity. If $p=q=0, x=-1$, and $N-m^{\star}-\bar{m}$ is even, the result is easily verified by direct computation. If $x=1$, all terms have the same sign. Set $m=\bar{m}+m^{\star}$. For sufficiently large $N$, the absolute value of each of the approximately $(N-m) / 2$ terms in the range $[(N-m) / 4] \leq n \leq 3[(N-m) / 4]$ is bounded below by $((N-m) / 4)^{p+q}$. The result follows.

As an immediate corollary of Lemma 4.6, or directly, we have the following.

Lemma 4.7. Let $\mu \in(0,1), p \in \mathbb{N}$. There exists $C=C(\mu, p)>0$, independent of $N$, such that

$$
\sum_{n=0}^{N}\left(\begin{array}{l}
n \\
p
\end{array}\right) \mu^{n} \beta_{N} \leq C \beta_{N} .
$$

4.4. Construction of a sequence of periodic points converging to $x_{H}$. For $N$ sufficiently large, we will construct a sequence $\left(\mathbf{p}_{N}\right) \subset K$ of periodic points converging to $x_{H}$. The point $\mathbf{p}_{N}$ will be of prime period $N$. Our construction depends on the construction of a pseudo-orbit followed by an application of the Anosov closing lemma. We present the proof first on the basis of our assumption that $\Phi$ is linear when restricted to the stable and unstable manifolds of $p$. This assumption of linearizability is removed in $\S 4.9$.

Since $\Phi$ is $C^{2}$, our assumption on the linearity of $\Phi$ restricted to the stable and unstable manifolds of $p$ allows for nonlinearities of the form $a_{i j}(\mathbf{x}, \mathbf{y}) x_{i} y_{j}$, where $a_{i j}$ is continuous.

Set $\Phi=\left(\Phi_{s}, \Phi_{u}\right)$. Since we are assuming $\Phi\left|\mathbb{E}^{s}=G_{s}, \Phi\right| \mathbb{E}^{u}=G_{u}$, it follows that on $K$ we may write

$$
\begin{aligned}
& \Phi_{s}(\mathbf{x}, \mathbf{y})=G_{s}(\mathbf{x})+\sum_{i, j} x_{i} y_{j} a_{i j}(\mathbf{x}, \mathbf{y}) \\
& \Phi_{u}(\mathbf{x}, \mathbf{y})=G_{u}(\mathbf{y})+\sum_{i, j} x_{i} y_{j} b_{i j}(\mathbf{x}, \mathbf{y})
\end{aligned}
$$

where $a_{i j}, b_{i k}$ are continuous.

Proposition 4.8. There exist $\bar{N} \in \mathbb{N}, C>0$, such that for $N \geq \bar{N}$, there exists a point $\mathbf{p}_{N} \in W$ of prime period $N$, such that if we set $\Phi^{n}\left(\mathbf{p}_{N}\right)=\mathbf{p}_{N}^{n}=\left(\mathbf{x}^{n}, \mathbf{y}^{n}\right)$, then

(1) $\mathbf{x}_{i}^{n}=J_{i}^{n}\left(\mathbf{A}_{i}\right)+\mathbf{C}_{i}^{n} \beta_{N}$;

(2) $\mathbf{y}_{j}^{n}=J_{j}^{n-N}\left(\mathbf{B}_{j}\right)+\mathbf{C}_{j}^{n} \beta_{N}$;

(3) $\left(\mathbf{p}_{N}^{n}\right) \subset K$;

where $\left\|\mathbf{C}_{i}^{n}\right\|,\left\|\mathbf{C}_{j}^{n}\right\| \leq C$, for all $n \in\{0, \ldots, N\}$. 
Proof. Given $N>0$, define for $n \in\{0, \ldots, N\}, \tilde{\mathbf{z}}^{n}=\left(\tilde{\mathbf{x}}^{n}, \tilde{\mathbf{y}}^{n}\right)$, where $\tilde{\mathbf{x}}_{i}^{n}=J_{i}^{n}\left(\mathbf{A}_{i}\right)$, $\tilde{\mathbf{y}}_{j}^{n}=J_{j}^{n-N}\left(\mathbf{B}_{j}\right)$. Certainly there exists $\bar{N}>0$ such that for $N \geq \bar{N},\left(\tilde{\mathbf{z}}^{n}\right)$ is well defined as a subset of $K$. A routine computation, using Lemmas 4.7 and 4.1 and equations (4.6) and (4.7), shows that there exists $\bar{C}>0$, independent of $N$, such that for all $N \geq \bar{N}$ we have $\left\|\Phi\left(\tilde{\mathbf{z}}^{n}\right)-\tilde{\mathbf{z}}^{n+1}\right\| \leq \bar{C} \beta_{N}$. We give the computation in the case when the matrix $G=D \Phi(0)$ is semisimple. Under this assumption, we have $\tilde{x}_{\ell}=\mu_{\ell}^{n} A_{\ell}, \tilde{y}_{k}=\lambda_{k}^{n-N} B_{k}$ and so, using (4.6), we have

$$
\begin{aligned}
\Phi\left(\tilde{\mathbf{z}}^{n}\right)_{i} & =\mu_{i}^{n+1} A_{i}+\sum_{\ell, k} \tilde{x}_{\ell} \tilde{y}_{k} a_{\ell k}\left(\tilde{\mathbf{z}}^{n}\right), \\
\Phi\left(\tilde{\mathbf{z}}^{n}\right)_{j} & =\lambda_{j}^{n+1-N} B_{j}+\sum_{\ell, k} \tilde{x}_{\ell} \tilde{y}_{k} b_{\ell k}\left(\tilde{\mathbf{z}}^{n}\right) .
\end{aligned}
$$

Shrinking $K$ if necessary, let $C^{\prime}=\max _{\ell, k}\left(\left\|a_{\ell k}\right\|_{0},\left\|b_{\ell k}\right\|_{0}\right)$, where the $C^{0}$ norms are computed on $K$. We have

$$
\begin{aligned}
\left|\sum_{\ell, k} \tilde{x}_{\ell} \tilde{y}_{k} a_{\ell k}\left(\tilde{\mathbf{z}}^{n}\right)\right| & \leq C^{\prime} \sum_{\ell, k}\left|A_{\ell} \| B_{k}\right|\left|\mu_{\ell}\right|^{n}\left|\lambda_{k}\right|^{n-N}, \\
& \leq C^{\prime} D \sum_{\ell, k} \Psi^{n} \Psi^{N-n} \quad \text { where } D=\max _{\ell, k}\left|A_{\ell} \| B_{k}\right|, \\
& \leq C^{\prime} D \Psi^{N} \sum_{\ell, k} 1, \\
& \leq C_{i} \Psi^{N} \leq C_{i} \beta_{N},
\end{aligned}
$$

where $C_{i}$ is independent of $N$. We have a similar estimate on $\sum_{\ell, k} \tilde{x}_{\ell} \tilde{y}_{k} b_{\ell k}\left(\tilde{\mathbf{z}}^{n}\right)$. In the case $n=N$, we use the (smooth) identification between $\tilde{W}$ and $W$ and our estimates on $\left\|\tilde{\mathbf{z}}^{N}-\tilde{x}_{H}\right\|,\left\|\tilde{\mathbf{z}}^{0}-x_{H}\right\|$, to deduce that $\left\|\tilde{\mathbf{z}}^{N}-\tilde{\mathbf{z}}^{0}\right\| \leq \bar{C} \beta_{N}$.

Since $X$ is hyperbolic, we may now apply the Anosov closing lemma [15, §6.4] to deduce that for a possibly larger value of $\bar{N}$, there exists $\hat{C}>0$, such that for $N \geq \bar{N}$, there exists a point $\mathbf{p}_{N} \in W$ of prime period $N$ such that for $n \in\{0, \ldots, N\}$, $\left\|\mathbf{p}_{N}^{n}-\tilde{\mathbf{z}}^{n}\right\| \leq \hat{C} \bar{C} \beta_{N}$.

COROLlary 4.9. Given $m \in \mathbb{N}$, there exists $C_{1}>0$ such that for all $N \geq \bar{N}$, $n \in\{0, \ldots, m\}$ we have

$$
\left\|\mathbf{p}_{N}^{n}-\mathbf{A}^{n}\right\|,\left\|\mathbf{p}_{N}^{N-n}-\mathbf{B}^{n}\right\| \leq C_{1} \beta_{N} .
$$

Proof. When $m=0$, the estimate follows with $C_{1}=2 C$ by Proposition 4.8. For $m>0$, we obtain the result by applying the mean value theorem to $\Phi^{n}, \Phi^{n-N}$.

Remarks 4.10. (1) The extension of Proposition 4.8 to allow for the case where $G$ has complex eigenvalues is immediate-indeed, the formalism already allows for complex eigenvalues.

(2) Since the original $H$-set $X$ is locally maximal, the periodic points given by Proposition 4.8 lie in $X$. 
(3) If we assume that $\Phi$ is $C^{1}$-linearizable at $p$, we can give somewhat sharper estimates on $\mathbf{p}_{N}^{n}$. Specifically, in the linearizing coordinates, the relations $\Phi\left(\mathbf{p}_{N}^{n}\right)=\mathbf{p}_{N}^{n+1}, 0 \leq n \leq$ $N-1$, imply that $\mathbf{x}_{i}^{n}=J_{i}^{n}\left(\mathbf{A}_{i}+\mathbf{C}_{i} \beta_{N}\right), \mathbf{y}_{j}^{n}=J_{j}^{n-N}\left(\mathbf{B}_{j}+\mathbf{C}_{j} \beta_{N}\right)$, where the constants $\mathbf{C}_{i}$, $\mathbf{C}_{j}$ are independent of $n$. It follows that if we define $\Theta=\max \left(\left|\mu_{S}\right|,\left|\lambda_{T}\right|^{-1}\right)+\varepsilon$, where $\varepsilon>0$ is chosen sufficiently small so that $\Theta<1$, then we can write

$$
\mathbf{x}_{i}^{n}=J_{i}^{n}\left(\mathbf{A}_{i}\right)+\mathbf{C}_{i}^{n} \Theta^{n} \beta_{N}, \quad \mathbf{y}_{j}^{n}=J_{j}^{n-N}\left(\mathbf{B}_{j}\right)+\mathbf{C}_{j}^{n} \Theta^{N-n} \beta_{N} .
$$

If $\Phi$ is $C^{2}$-linearizable at $p$, then these estimates yield simpler proofs of some of our lemmas (notably Lemmas 4.13 and 4.14). However, the results of these lemmas may fail if $\Phi$ is not $C^{2}$-linearizable at $p$, and the full proof of Theorem 2.10 requires some special arguments to take account of this. For ease of exposition, we assume $C^{2}$-linearizability wherever it is helpful to do so, and sketch the general case at the end of this sectionsee $\$ 4.9$.

Remark 4.11. From now on we identify a neighborhood $U$ of the closure of the homoclinic orbit of $x_{H}$ in $M$ with $\hat{K} \cup W$ in $\mathbb{R}^{n}$.

Therefore, functions $F$ defined on $\mathbb{R}^{n}$ induce functions on $U \subset M$ through their restriction $F \mid(\hat{K} \cup W)$. When we compute the average of $F$, we use the representatives of $\mathbf{p}_{N}$ and $x_{H}$ situated in $W$, that is, near $(\mathbf{A}, 0)$.

4.5. Upper bounds. We continue to assume that $\Phi$ is linear when restricted to the local stable and unstable manifolds at $p$-this assumption is relaxed in $\$ 4.9$. Throughout this and the following subsection ' $c$ ', ' $C$ ' will always denote constants independent of $N \geq \bar{N}$ and cocycles $F$.

Let $\left(\mathbf{p}_{N}\right)_{N \geq \bar{N}}$ be the sequence of periodic points given by Proposition 4.8. Recall that we defined $A_{N}(F)=\operatorname{Av}\left(F, x_{H}\right)-\operatorname{Av}\left(F, \mathbf{p}_{N}\right)$ in $\S 3$.

LEMMA 4.12. Let $r \geq 2$. There exists $C>0$ such that if $F \in C^{r}\left(\mathbb{R}^{m}, \mathbb{R}\right)$ with $F(0)=0$ and $D F(0)=0$, then

$$
\left|A_{N}(F)\right| \leq C \gamma_{N}\|F\|_{2} \quad \text { for all } N \geq \bar{N}
$$

Proof. We assume that $G=D \Phi(0)$ is semisimple with real eigenvalues. In particular, $\beta_{N}=\Psi^{N}$ and either $\gamma_{N}=\Psi^{N}$ or $\gamma_{N}=N \Psi^{N}$ ( $\Psi$-resonance). The proof in the case when $G$ has complex eigenvalues or is non-semisimple is essentially the same, just longer (note that when we consider the more difficult problem of finding lower bounds we do allow for the non-semisimple case).

It follows from Taylor's theorem that we may write

$$
F(x, y)=\sum_{i} x_{i} L_{i}(x)+\sum_{i, \ell} x_{i} y_{\ell} M_{i \ell}(x, y)+\sum_{\ell} y_{\ell} N_{\ell}(y),
$$

where $L_{i}, N_{\ell}$ are $C^{1}$ and vanish at the origin, and $M_{i \ell}$ is continuous. 
We start by estimating the second term in this sum. We have $\operatorname{Av}\left(x_{i} y_{\ell} M_{i \ell}, x_{H}\right)=0$ and so it suffices to estimate $\operatorname{Av}\left(x_{i} y_{\ell} M_{i \ell}, \mathbf{p}_{N}\right)$. We have

$$
\begin{aligned}
\left|\operatorname{Av}\left(x_{i} y_{\ell} M_{i \ell}, \mathbf{p}_{N}\right)\right| & =\left|\sum_{n=0}^{N-1} x_{i}^{n} y_{\ell}^{n} M_{i \ell}\left(\mathbf{p}_{N}^{n}\right)\right|, \\
& \leq\left\|M_{i \ell}\right\|_{0}\left|\sum_{n=0}^{N-1}\left(\mu_{i}^{n} A_{i}+c_{i}^{n} \beta_{N}\right)\left(\lambda_{\ell}^{n-N} B_{\ell}+c_{\ell}^{n} \beta_{N}\right)\right|, \\
& \leq\left\|M_{i \ell}\right\|_{0}\left|\sum_{n=0}^{N-1} \mu_{i}^{n} \lambda_{\ell}^{n} \lambda_{\ell}^{-N} A_{i} B_{\ell}+c_{1} \beta_{N}\left(\left|\mu_{i}\right|^{n}+\left|\lambda_{\ell}\right|^{n-N}\right)+c_{2} \beta_{N}^{2}\right|, \\
& \leq\left\|M_{i \ell}\right\|_{0}\left(\left|\sum_{n=0}^{N-1} \mu_{i}^{n} \lambda_{\ell}^{n} \lambda_{\ell}^{-N} A_{i} B_{\ell}\right|+|c| \beta_{N}\right) .
\end{aligned}
$$

If $\mu_{i} \lambda_{\ell} \neq 1$ and we ignore trivial cases where $A_{i} B_{\ell}=0$, then

$$
\left|\sum_{n=0}^{N-1} \mu_{i}^{n} \lambda_{\ell}^{n} \lambda_{\ell}^{-N}\right|=\left|\frac{\lambda_{\ell}^{-N}-\mu_{i}^{N}}{1-\mu_{i} \lambda_{\ell}}\right| \leq c \Psi^{N}=c \beta_{N}=c \gamma_{N} .
$$

If $\mu_{i} \lambda_{\ell}=1$, then $\sum_{n=0}^{N-1} \mu_{i}^{n} \lambda_{\ell}^{n} \lambda_{\ell}^{-N}=N \lambda_{\ell}^{-N}$. In the case $\left|\lambda_{\ell}\right|^{-1}=\Psi$ we have the estimate $\left|\sum_{n=0}^{N-1} \mu_{i}^{n} \lambda_{\ell}^{n} \lambda_{\ell}^{-N}\right| \leq c N \Psi^{N}$, otherwise we bound by $c \Psi^{N}$. Using our estimates, it follows that $\left|\operatorname{Av}\left(x_{i} y_{\ell} M_{i \ell}, \mathbf{p}_{N}\right)\right| \leq c\left\|M_{i \ell}\right\|_{0} \gamma_{N}$, and hence

$$
\left|\operatorname{Av}\left(x_{i} y_{\ell} M_{i \ell}, \mathbf{p}_{N}\right)\right| \leq c \gamma_{N}\|F\|_{2}
$$

Summing over $i, \ell$, we obtain the required estimate.

We conclude by estimating the first sum. We have

$$
\begin{aligned}
A_{N}\left(x_{i} L_{i}\right) \leq & \left|\sum_{n=0}^{N-1}\left(\mu_{i}^{n} A_{i}+c_{i}^{n} \beta_{N}\right) L_{i}\left(\mu_{i}^{n} A_{i}+c_{i}^{n} \beta_{N}\right)-\mu_{i}^{n} A_{i} L_{i}\left(\mu_{i}^{n} A_{i}\right)\right| \\
& +\sum_{n=N}^{\infty}\left|\mu_{i}^{n} A_{i} L_{i}\left(\mu_{i}^{n} A_{i}\right)\right| \\
\leq & \left|\sum_{n=0}^{N-1}\left(\mu_{i}^{n} A_{i}+c_{i}^{n} \beta_{N}\right)\left(L_{i}\left(\mu_{i}^{n} A_{i}\right)+c\left\|L_{i}\right\|_{1} \beta_{N}\right)-\mu_{i}^{n} A_{i} L_{i}\left(\mu_{i}^{n} A_{i}\right)\right| \\
& +c\left|A_{i}\right| \Psi^{N}\left\|L_{i}\right\|_{0}, \\
\leq & c\left\|L_{i}\right\|_{1} \beta_{N} \leq c\|F\|_{2} \gamma_{N},
\end{aligned}
$$

where we have used $\left|L_{i}\left(\mu_{i}^{n} A_{i}\right)\right| \leq\left\|L_{i}\right\|_{1}\left|\mu_{i}^{n} A_{i}\right|$.

Lemma 4.13. Fix $r>0$. Assume that $\Phi$ is $C^{2}$-linearizable at $p$ if $r \geq 2$. There exists $C>0$, such that for all $F \in C^{r}\left(\mathbb{R}^{m}, \mathbb{R}\right)$ with $F(0)=0$ we have

(1) if $0<r<2$,

$$
\left|A_{N}(F)\right| \leq C \bar{\beta}_{N}(r)\|F\|_{r} \quad \text { for all } N \geq \bar{N}
$$

(2) if $r \geq 2$,

$$
\left|A_{N}(F)\right| \leq C \gamma_{N}\|F\|_{2} \quad \text { for all } N \geq \bar{N}
$$


Proof. First of all we take $r \geq 2$. Again, we give the details only for the case when $G$ is semisimple and eigenvalues are real. Without loss of generality, we may make a preliminary $C^{2}$-linearizing change of coordinates. Since $F$ is $C^{2}$, we may find $a_{i} \in$ $C^{1}\left(\mathbb{E}^{s}, \mathbb{R}\right), b_{j} \in C^{1}\left(\mathbb{E}^{u}, \mathbb{R}\right)$ and $H_{i j} \in C^{0}\left(\mathbb{R}^{m}, \mathbb{R}\right)$ such that

$$
F(\mathbf{x}, \mathbf{y})=\sum_{i=1}^{S} x_{i} a_{i}(\mathbf{x})+\sum_{j=1}^{T} y_{j} b_{j}(\mathbf{y})+\sum_{i=1}^{S} \sum_{j=1}^{T} x_{i} y_{j} H_{i j}(\mathbf{x}, \mathbf{y}) .
$$

It follows from Lemma 4.12 that we can reduce to the case where

$$
F(\mathbf{x}, \mathbf{y})=\sum_{i=1}^{S} x_{i} a_{i}+\sum_{j=1}^{T} y_{j} b_{j}
$$

and $a_{i}, b_{j} \in \mathbb{R}$. It follows from Proposition 4.8 and Remark 4.10(3) that $x_{i}^{n}=A_{i} \mu_{i}^{n}+$ $c_{i}^{n} \Theta^{n} \Psi^{N}, 1 \leq i \leq S$, where the $\left(c_{i}^{n}\right)$ are uniformly bounded and $0<\Theta<1$. Fixing $i$, we have $\left|A_{N}\left(x_{i} a_{i}\right)\right| \leq E_{1}+E_{2}$, where

$$
E_{1}=\left|\sum_{n=0}^{N-1}\left(\mu_{i}^{n} A_{i}+c_{i}^{n} \Theta^{n} \Psi^{N}\right) a_{i}-\mu_{i}^{n} A_{i} a_{i}\right|, \quad E_{2}=\left|\sum_{n=N}^{\infty} \mu_{i}^{n} A_{i} a_{i}\right| .
$$

Estimates on $E_{1}, E_{2}$ are trivial if $A_{i}=0$. Therefore, we suppose in what follows that $A_{i} \neq 0$ and so $\left|\mu_{i}\right| \leq \Psi$. Then,

$$
\begin{array}{r}
E_{1}=\left|a_{i}\right|\left|\sum_{n=0}^{N-1} c_{i}^{n} \Theta^{n} \Psi^{N}\right| \leq c\|F\|_{2} \Psi^{N} \leq c \gamma_{N}\|F\|_{2}, \\
E_{2}=\left|\sum_{n=N}^{\infty} \mu_{i}^{n} A_{i} a_{i}\right| \leq c\left|a_{i}\left\|\left.\mu_{i}\right|^{N} \leq c \gamma_{N}\right\| F \|_{2} .\right.
\end{array}
$$

Summing over $i$, we have shown $\left|A_{N}\left(x_{i} a_{i}\right)\right| \leq c \gamma_{N}\|F\|_{2}$. A completely analogous argument handles the term $\sum_{j=1}^{T} y_{j} b_{j}$.

It remains to consider what happens if $0<r<2$ (still assuming that $\Phi$ is linear restricted to the stable and unstable manifolds of $p$ ). We allow for $\tilde{\mu}$ and $\tilde{\lambda}$ to be nonsimple eigenvalues.

On $\hat{K} \cup W$ we may write $F(x, y)=L(x)+M(y)+H(x, y)$, where $L(x)=F(x, 0)$, $M(y)=F(0, y)$ and $H(x, y)=F(x, y)-L(x)-M(y)$. Necessarily, $H$ vanishes on $\mathbb{E}^{s}, \mathbb{E}^{u}$ in $\hat{K} \cup W$. If $0<r \leq 1$, then, we may use the obvious Hölder estimates on $L$ and the estimates of Proposition 4.8 to show that $\left|A_{N}(L)\right| \leq c \bar{\beta}_{N}(r)\|L\|_{r}$. Similarly for $M$. This leaves the term $H(x, y)$. Since $\operatorname{Av}\left(H, x_{H}\right)=0$, we must estimate $\left|\operatorname{Av}\left(H, \mathbf{p}_{N}\right)\right|$. For $0 \leq n \leq N$, we may write $H\left(\mathbf{p}_{N}^{n}\right)=H\left(\mathbf{p}_{N}^{n}\right)-H\left(\mathbf{x}_{N}^{n}\right)=H\left(\mathbf{p}_{N}^{n}\right)-H\left(\mathbf{y}_{N}^{n}\right)$. We have the estimates

$$
\left|H\left(\mathbf{p}_{N}^{n}\right)-H\left(\mathbf{x}_{N}^{n}\right)\right| \leq\|H\|_{r}\left\|\mathbf{y}_{N}^{n}\right\|^{r}, \quad\left|H\left(\mathbf{p}_{N}^{n}\right)-H\left(\mathbf{y}_{N}^{n}\right)\right| \leq\|H\|_{r}\left\|\mathbf{x}_{N}^{n}\right\|^{r} .
$$

In order to get the optimal estimate, we switch from $x$ - to $y$-coordinates at the value $N_{c}$ of $n$ for which $\left\|\mathbf{x}_{N}^{n}\right\| \approx\left\|\mathbf{y}_{N}^{n}\right\|$. A straightforward computation shows that if $\Psi=\tilde{\mu}$ then

$$
N_{c}=\frac{\alpha N}{1+\alpha}-\frac{p-q}{1+\alpha} \frac{\ln N}{\ln \Psi}+O(1)
$$


where $\alpha=\min \{-\ln |\tilde{\lambda}|, \ln |\tilde{\mu}|\} / \ln \Psi$. It follows easily that we can choose $\Theta \in(\Psi, 1)$ and a constant $C>0$ such that $\left\|\mathbf{x}_{N}^{n}\right\| \leq C \Theta^{n} \bar{\beta}_{N}, n \geq N_{c}$. We have a similar estimate on $\left\|\mathbf{y}_{N}^{n}\right\|$, $n<N_{c}$. This suffices to obtain the estimate $\left|\operatorname{Av}\left(H, \mathbf{p}_{N}\right)\right| \leq c \bar{\beta}_{N}(r)\|H\|_{r}$. The analysis in the case $\Psi=\tilde{\lambda}^{-1}$ is similar.

Finally, suppose $r \in(1,2)$. We write $F=L+M+H$ as before, and assume $\Psi=\tilde{\mu}$. The estimates on $L$ and $M$ are straightforward and we omit details. It remains to consider the term $H(x, y)$. Noting that $H$ vanishes on the $x$ - and $y$-axes, and applying Taylor's theorem with integral remainder, we have

$H(x, y)=\left\{\begin{array}{l}D_{y} H(x, 0)(y)+\int_{0}^{1}\left[D_{y} H(x, t y)-D_{y}(x, 0)\right](y) d t=A(x)(y)+I_{1}(x, y) \\ D_{x} H(0, y)(x)+\int_{0}^{1}\left[D_{y} H(t x, y)-D_{y}(0, y)\right](x) d t=B(y)(x)+I_{2}(x, y),\end{array}\right.$

where $A(x), B(y)$ are linear maps depending $C^{r-1}$ on $x$ and $y$, respectively, The integral terms are estimated by $\left|I_{1}(x, y)\right| \leq\|H\|_{r}\|y\|^{r}$ and $\left|I_{2}(x, y)\right| \leq\|H\|_{r}\|x\|^{r}$. Since $A$ and $B$ vanish at the origin, we also have the estimates $|A(x)(y)| \leq\|H\|_{r}\|x\|^{r-1}\|y\|$ and $|B(y)(x)| \leq\|H\|_{r}\|y\|^{r-1}\|x\|$. Just as in the first part of the proof, we switch from one expansion to the other at $n=N_{c}$, and bound using the above estimates on $\left|I_{1}(x, y)\right|,\left|I_{2}(x, y)\right|,|A(x)(y)|$ and $|B(y)(x)|$. The sum of the $\|x\|\|y\|^{r-1}$ terms involves the exponent $n(1-\alpha(r-1))$, its sign determining the three possible outcomes in the definition of $\bar{\beta}_{N}(r)$. We omit the straightforward details.

4.6. Lower bounds for functions in the class $C^{r}, r \geq 2$. We continue to assume that $\Phi$ is linear when restricted to the local stable and unstable manifolds at $p$. In this subsection we construct an explicit $C^{\infty}$-cocycle $F$ for which $\left|A_{N}(F)\right|$ decays at the slowest possible rate for $C^{2}$-functions. (The lower bound obtained in this subsection is not optimal when $r<2$.)

Lemma 4.14. Let $D \subset K$ be an open neighborhood of $p$. There exists $F \in C^{\infty}\left(\mathbb{R}^{m}, \mathbb{R}\right)$ with $\operatorname{supp}(F) \subset D$ and $C>0$ such that for infinitely many values of $N$ we have

$$
\left|A_{N}(F)\right| \geq C \gamma_{N}
$$

Proof. For the proof we assume the notation of $\S 4.2$. Let $\bar{m}, m^{\star} \in \mathbb{N}$, and define $O^{\star}\left(x_{H}\right)=\left\{\Phi^{n}\left(x_{H}\right) \mid n \in\left(-m^{\star}, \bar{m}\right)\right\}$ and $O_{m}\left(x_{H}\right)=O\left(x_{H}\right) \backslash O^{\star}\left(x_{H}\right)$. For $\bar{m}, m^{\star}$ sufficiently large, we may choose disjoint compact neighborhoods $U_{m}, V_{m}$ of $\overline{O_{m}\left(x_{H}\right)}$, $O^{\star}\left(x_{H}\right)$ respectively so that $U_{m} \subset D, V_{m} \subset \hat{K} \backslash D$. We remark that having chosen $\bar{m}, m^{\star}$, we can find $N^{\star}=N\left(\bar{m}, m^{\star}\right) \geq \bar{N}$ such that for all $N \geq N^{\star}, \mathbf{p}_{N}^{n} \in U_{m}, n \notin\left(-m^{\star}, \bar{m}\right)$ and $\mathbf{p}_{N}^{n} \in V_{m}, n \in\left(-m^{\star}, \bar{m}\right)$. In the sequel we shall assume this without further comment.

Define $F=F_{\bar{m}, m^{\star}} \in C^{\infty}\left(\mathbb{R}^{m}, \mathbb{R}\right)$ by

$$
F(\mathbf{x}, \mathbf{y})= \begin{cases}0, & (\mathbf{x}, \mathbf{y}) \notin D, \\ x_{\bar{l} 1} y_{\bar{J} 1}, & (\mathbf{x}, \mathbf{y}) \in U_{m} .\end{cases}
$$

We show that we can choose $\bar{m}, m^{\star}$ so that $F$ satisfies the conditions of the lemma. 
Although in the proof we allow $G$ to have non-trivial Jordan blocks, we simplify a little by assuming that the dominant eigenvalues $\mu=\mu_{\bar{i}}, \lambda=\lambda_{\bar{j}}$ are real. (If dominant eigenvalues are complex, we work with a $\mathbb{C}$-valued cocycle $F$ and obtain the required estimates by taking the real part of $F$.)

Since $F$ vanishes identically on $\mathbb{E}^{s}, \mathbb{E}^{u}$, it follows that it suffices to find $C>0, \hat{N} \geq \bar{N}$, $m \in \mathbb{N}$ such that for $N \geq \hat{N}$,

$$
\left|\operatorname{Av}\left(F, \mathbf{p}_{N}\right)\right| \geq C \gamma_{N} .
$$

As usual, we write $\mathbf{p}_{N}^{n}=\left(\mathbf{x}^{n}, \mathbf{y}^{n}\right)$ and set $\mathbf{x}^{n}=\left(\mathbf{x}_{1}^{n}, \ldots, \mathbf{x}_{s}^{n}\right), \mathbf{y}^{n}=\left(\mathbf{y}_{1}^{n}, \ldots, \mathbf{y}_{t}^{n}\right)$. We need to compute the $(\bar{l}, 1)$-component of $G_{s}^{n}\left(\mathbf{x}^{0}\right)$ and the $(\bar{J}, 1)$-component of $G_{u}^{n}\left(\mathbf{y}^{0}\right)$, $0 \leq n \leq N$.

Set $\mu=\mu_{\bar{\imath}}, \lambda=\lambda_{\bar{j}}$ and $\bar{m}=m(\bar{l})>0$. We have

$$
\mathbf{x}_{\bar{l}}^{\bar{m}}=\mathbf{A}_{\bar{l}}^{\bar{m}}+\mathbf{C}_{\bar{l}}^{\bar{m}} \beta_{N}
$$

where $\left\|\mathbf{C}_{\bar{l}}^{\bar{m}}\right\| \leq C$, independent of $m, n, N$, and $\mathbf{A}_{\underline{l} 1}^{n} \neq 0$, for all $n \geq \bar{m}$. (In future we drop the sub- and superscripts from the constants $\mathbf{C}_{\bar{l}}^{\bar{m}}$.) It follows from Lemma 4.1 and the definition of $p$ that for $N \geq n \geq \bar{m}$, we have

$$
\begin{aligned}
\mathbf{x}_{\bar{l} 1}^{n} & =\sum_{i=1}^{p}\left(\begin{array}{c}
n-\bar{m} \\
i-1
\end{array}\right) \mu^{n+1-i-\bar{m}} A_{\bar{\imath} i}^{\bar{m}}+\mathbf{C} \beta_{N}, \\
& =E_{1}^{\prime}+E_{2}^{\prime}
\end{aligned}
$$

where (Lemma 4.2)

$$
\left|A_{\bar{l} p}^{\bar{m}}\right| \geq C|\mu|^{\bar{m}}
$$

and $C$ is independent of $\bar{m}$. It follows from our choice of $\bar{m}=m(\bar{l})$, that $A_{\bar{l} 1}^{\bar{m}} \neq 0$ and hence the dominant term in the sum for $E_{1}^{\prime}$ is $A_{\bar{l} p}^{\bar{m}}\left(\begin{array}{c}n \\ p-1\end{array}\right) \mu_{\bar{l}}^{n+1-p}$. Increasing $\bar{m}$ if necessary, it follows from the estimates of Lemma 4.1 that for $\bar{m} \leq n \leq N$,

$$
E_{1}^{\prime}=A_{\bar{\imath} p}^{\bar{m}}\left(\begin{array}{c}
n-\bar{m} \\
p-1
\end{array}\right) \mu_{\bar{\imath}}^{n-\bar{m}+1-p}\left(1+k_{n}\right)
$$

where $\left|k_{n}\right| \leq 0.1$. Notice that we only improve this estimate if we further increase $\bar{m}$ working always within the requirement that $\bar{m} \leq n \leq N$.

On the other hand, for all $\bar{m} \leq n \leq N,\left|E_{2}^{\prime}\right|$ is bounded above by $C \beta_{N}$. Combining our estimates for $E_{1}^{\prime}, E_{2}^{\prime}$, it follows that for $\bar{m} \leq n \leq N$ we have

$$
\mathbf{x}_{\bar{l} 1}^{n}=A_{\bar{\imath} p}^{\bar{m}}\left(\begin{array}{c}
n-\bar{m} \\
p-1
\end{array}\right) \mu_{\bar{l}}^{n-\bar{m}+1-p}\left(1+k_{n}\right)+C \beta_{N} .
$$

We may similarly choose $m^{\star} \in \mathbb{N}$ such that for $0 \leq n \leq N-m^{\star}$,

$$
\mathbf{y}_{\bar{\jmath} 1}^{n}=B_{\bar{\jmath} q}^{m^{\star}}\left(\begin{array}{c}
-N+n+m^{\star} \\
q-1
\end{array}\right) \lambda^{-N+n+m^{\star}-q+1}\left(1+k_{n}^{\star}\right)+C^{\star} \beta_{N},
$$

where $\left|k_{n}^{\star}\right| \leq 0$.1. Again, we only improve estimates by increasing $m^{\star}$. We also have the estimate

$$
\left|B_{\bar{l} q}^{m^{\star}}\right| \geq C^{\star}|\lambda|^{-m^{\star}}
$$


Increasing $\bar{m}, m^{\star}$ will not change any of the previous estimates as all constants $C, C^{\star}$ are independent of $\bar{m}, m^{\star}$.

Set $K^{\star}=A_{\bar{l} p}^{\bar{m}} B_{\bar{\jmath} q}^{m^{\star}} \neq 0$. Noting our definition of $F_{\bar{m}, m^{\star}}$, and relabeling the error terms $k, k^{\star}$, we see that

$$
\begin{aligned}
\operatorname{Av}\left(F_{\bar{m}, m^{\star}}, \mathbf{p}_{N}\right) & =\sum_{n=\bar{m}}^{N-m^{\star}} \mathbf{x}_{\bar{l} 1}^{n} \mathbf{y}_{\bar{\jmath} 1}^{n}, \\
& =E_{1}+E_{2}+E_{3}+E_{4},
\end{aligned}
$$

where

$$
\begin{aligned}
& E_{1}=K^{\star} \sum_{n=\bar{m}}^{N-m^{\star}}\left(\begin{array}{c}
n-\bar{m} \\
p-1
\end{array}\right)\left(\begin{array}{c}
-N+m^{\star}+n \\
q-1
\end{array}\right) \mu^{n+1-\bar{m}-p} \lambda^{-N+m^{\star}+n-q+1}\left(1+k_{n}\right)\left(1+k_{n}^{\star}\right), \\
& \left|E_{2}\right| \leq|C|\left|B_{\bar{\jmath} q}^{m^{\star}}\right| \beta_{N} \sum_{n=\bar{m}}^{N-m^{\star}}\left(\begin{array}{c}
n-\bar{m} \\
p-1
\end{array}\right)|\mu|^{n+1-\bar{m}-p}\left(1+k_{n}\right), \\
& \left|E_{3}\right| \leq\left|C^{\star}\right|\left|A_{\bar{l} p}^{\bar{m}}\right| \beta_{N} \sum_{n=\bar{m}}^{N-m^{\star}}\left(\begin{array}{c}
-N+m^{\star}+n \\
q-1
\end{array}\right)|\lambda|^{-N+m^{\star}+n-q+1}\left(1+k_{n}^{\star}\right), \\
& \left|E_{4}\right| \leq|C|\left|C^{\star}\right| N \beta_{N}^{2} .
\end{aligned}
$$

Suppose, first, that we are in the non-resonant case and $G=D \Phi(0)$ is semisimple. Without loss of generality, we take $\Psi=|\lambda|^{-1} \geq|\mu|$ and so $|\lambda \mu| \leq 1$ (if $\lambda \mu=-1$, we take $N-\bar{m}-m^{\star}$ to be even). It follows from (4.10) and (4.11) that $\left|K^{\star}\right| \geq C|\lambda|^{-m^{\star}}|\mu|^{\bar{m}}$. Substituting in our expression for $E_{1}$, it follows (since $|\lambda \mu| \leq 1$ ) that

$$
\left|E_{1}\right| \geq c \Psi^{N}|\lambda \mu|^{\bar{m}}
$$

where $c>0$ is independent of $m, N$. On the other hand, for large enough $N$, we find that

$$
\begin{aligned}
& \left|E_{2}\right| \leq C_{2}|\lambda|^{-m^{\star}} \Psi^{N}, \\
& \left|E_{3}\right| \leq C_{3}|\mu|^{\bar{m}} \Psi^{N},
\end{aligned}
$$

where $C_{1}, C_{2}$ are independent of $\bar{m}, m^{\star}$, for large enough $N$. Since $|\lambda \mu|>|\mu|$, it is obvious that $\left|E_{1}\right| \gg\left|E_{3}\right|$ for sufficiently large $\bar{m}$. Fix such an $\bar{m}$. Now choose $m^{\star}$ large enough so that $C_{3}|\lambda|^{-m^{\star}} \ll c|\lambda \mu|^{\bar{m}}$. Hence $\left|E_{1}\right|$ dominates $\left|E_{2}\right|$ and $\left|E_{3}\right|$.

Since $\left|E_{1}\right|$ obviously dominates $\left|E_{4}\right|$ (Lemma 4.5), it follows that we can choose $\bar{m}, m^{\star}$, $C>0$, so that for all sufficiently large $N$ we have $\left|\operatorname{Av}\left(F_{\bar{m}, m^{\star}}, \mathbf{p}_{N}\right)\right| \geq C \Psi^{N}$ (note that if $\lambda \mu=1$, we choose the parity of $N$ so that the $E_{1}$ sum is non-vanishing!).

The proof in the resonant case is similar, the main difference being the appearance of the factor $N-\bar{m}-m^{\star}$ in the sum for $E_{1}$.

There remains the case when $G$ is not semisimple. There are three cases to consider: (1) $\Psi=|\mu|, \mu \lambda \neq 1$, (2) $\Psi=|\lambda|^{-1}, \mu \lambda \neq 1$, and (3) $\mu \lambda=1$. We consider case (1). (Details for the other cases are similar-simpler for the $\Psi$-resonant case-and make essential use of Lemma 4.6.) Suppose, then, that $\Psi=|\mu|$ and $|\lambda \mu|>1$. We have $\gamma_{N}=\beta_{N}=N^{p-1} \Psi^{N}$, where $p \geq 2$. Estimating $E_{1}, E_{2}, E_{3}$, using Lemma 4.6, we find 
constants $c, c^{\prime}, C_{2}, C_{3}>0$, independent of $\bar{m}, m^{\star}$ and sufficiently large $N$, such that

$$
\begin{aligned}
& \left|E_{1}\right| \geq c\left(N-\bar{m}-m^{\star}\right)^{p-1} \Psi^{N}|\lambda \mu|^{-m^{\star}} \geq c^{\prime} \beta_{N}|\lambda \mu|^{-m^{\star}}, \\
& \left|E_{2}\right| \leq C_{2}|\lambda|^{-m^{\star}} \beta_{N}, \\
& \left|E_{3}\right| \leq C_{3} \beta_{N}|\mu|^{\bar{m}} .
\end{aligned}
$$

First, we choose $m^{\star}$ sufficiently large so that $\left|E_{2}\right| \ll\left|E_{1}\right|$ for large $N$. Then fix $m^{\star}$ and choose $\bar{m}$ sufficiently large so that $\left|E_{3}\right| \ll\left|E_{1}\right|$ for large $N$. Since $\left|E_{1}\right|$ dominates $\left|E_{4}\right|$ for large $N$ (Lemma 4.5), the result follows.

4.7. Lower bounds for functions in the class $C^{r}, r<2$. In this subsection, for each $r \in(0,2)$ we construct an explicit $C^{r}$-cocycle $P_{r}$ for which $\left|A_{N}\left(P_{r}\right)\right|$ decays at the slowest possible rate for $C^{r}$-functions. We continue to assume that $\Phi$ is linear on the stable and unstable manifolds through $p$.

Lemma 4.15. Let $r \in(0,2)$. Let $D \subset K$ be an open neighborhood of $p$. There exists $P_{r} \in C^{r}\left(\mathbb{R}^{m}, \mathbb{R}\right) \backslash \bigcup_{s>r} C^{s}\left(\mathbb{R}^{m}, \mathbb{R}\right)$ and $C=C_{r}>0$ such that $\operatorname{supp}\left(P_{r}\right) \subset D$ and for infinitely many values of $N$ we have

$$
\left|A_{N}\left(P_{r}\right)\right| \geq C \bar{\beta}_{N}(r) .
$$

Proof. We shall also take $D=K$-the construction for general $D$ is analogous to that of Lemma 4.14.

Let $r \in(0,2)$. Define $P_{r}: \mathbb{R}^{m} \rightarrow \mathbb{R}$ by

$$
\begin{aligned}
P_{r}(\mathbf{x}, \mathbf{y}) & =0, \quad(\mathbf{x}, \mathbf{y}) \notin K, \text { or } x_{S}=y_{T}=0, \\
& =x_{S} y_{T} /\left(x_{S}^{2}+y_{T}^{2}\right)^{1-r / 2}, \quad(\mathbf{x}, \mathbf{y}) \in K .
\end{aligned}
$$

It is easy to verify that $P_{r}$ is of class $C^{r}, r \in(0,2)$. We claim that $\left|A_{N}\left(P_{r}\right)\right| \geq C \bar{\beta}_{N}(r)$. Since $P_{r}$ vanishes on the orbit of $x_{H}$, it suffices to estimate $\left|\operatorname{Av}\left(P_{r}, \mathbf{p}_{N}\right)\right|$.

Without loss of generality, suppose that $\Psi=\left|\mu_{S}\right| \geq\left|\lambda_{T}^{-1}\right|$. Let $\alpha=\ln \left|\lambda_{T}\right|^{-1} / \ln \Psi$. For simplicity we assume that both $\mu_{S}$ and $\lambda_{T}$ are real, positive and have algebraic multiplicity 1 . Details are similar in the general case. Estimating the sum, we find that

$$
\left|\operatorname{Av}\left(P_{r}, \mathbf{p}_{N}\right)\right|=(C+o(1)) \sum_{n=0}^{N-1} \frac{\Psi^{n} \Psi^{\alpha(N-n)}}{\left(a \Psi^{2 n}+b \Psi^{2 \alpha(N-n)}\right)^{1-r / 2}},
$$

where $C, a, b>0$. Estimating the term with $n=N_{c}$ (see equation (4.9) in the proof of Lemma 4.13) shows that $\left|\operatorname{Av}\left(P_{r}, \mathbf{p}_{N}\right)\right|$ is at least of order $\left(\Psi^{N}\right)^{r \alpha / 1+\alpha}=\left(\bar{\beta}_{N}\right)^{r}$, which proves the claim for $1-\alpha(r-1)>0$. To deal with the general case, break up the sum at $N_{c}$, factor out the leading term in the denominators, and compute the two sums.

4.8. Proof of Theorem 2.10. For $r \geq 2$ and $C^{2}$-linearizable $\Phi$, Theorem 2.10 follows immediately from the next result.

LEMMA 4.16. Suppose that $\Phi$ is $C^{2}$-linearizable at $p$. Let $r \geq 2$ and let $B=B^{r}(U, \mathbb{R})$, $L=L^{r}(U, \mathbb{R})$. Let $D^{\star} \subset U$ be a neighborhood of the closure of the $\Phi$-orbit of $x_{H}$ and $D \subset D^{\star}$ be a neighborhood of $p$. Let $F$ be the cocycle supported in D that was constructed in Lemma 4.14. Then: 
(1) $F \notin \overline{B+L}$;

(2) there is a $C^{2}$-neighborhood $\mathcal{W}$ of $F$ in $C^{r}(U, \mathbb{R})$ such that $F^{\prime} \notin \overline{B+L}, F^{\prime} \in \mathcal{W}$;

(3) if $H \in C^{r}(U, \mathbb{R})$ has $\operatorname{supp}(H) \cap D^{\star}=\emptyset$, then $F^{\prime}+H \notin \overline{B+L}, F^{\prime} \in \mathcal{W}$.

Proof. Choose $F$ as in Lemma 4.14. Then there exists a constant $C_{1}>0$ such that $\left|A_{N}(F)\right| \geq C_{1} \gamma_{N}$ for all sufficiently large $N$ (in the case $\lambda \mu=-1, p=q=1, N$ will either be odd or even). In particular, $A_{N}(F)$ is not eventually zero, so, by (3.4), $F \notin B+L$.

Now, let $g \in C^{r}(U, \mathbb{R})$ and choose $g$ so that $\|g\|_{2}<C_{1} / 2 C_{2}$ where $C_{2}$ is the constant in Lemma 4.13. It follows that

$$
\left|A_{N}(F+g)\right| \geq \frac{C_{1}}{2} \gamma_{N} .
$$

Again, $F+g \notin B+L$, proving statements (1) and (2) of the lemma. The final statement follows since $\operatorname{Av}\left(H, x_{H}\right)=0$ and $\operatorname{Av}\left(H, \mathbf{p}_{N}\right)=0$ for sufficiently large $N$. This concludes the proof of Lemma 4.16.

Remark 4.17. If $r \in(0,2)$ and $\Phi$ is linear on the stable and unstable manifolds through $p$, then Lemma 4.16 remains true with the proviso that the neighborhood $\mathcal{W}$ is now $C^{r}$-open and we no longer require the $C^{2}$-linearizability of $\Phi$ at $p$. The proof follows that of Lemma 4.16 except that we use Lemma 4.15 instead of Lemma 4.14, and $P_{r}$ instead of $F$.

4.9. The non-linearizable case. We conclude this section by sketching the approach we follow in the case when $\Phi$ is not $C^{2}$-linearizable. We begin by supposing that $\Phi$ is linear at least on the local stable and unstable manifolds of $p$. Hence, we may assume the notation and results of $\S 4.2$. We may suppose that $r \geq 2$, since the case $r<2$ did not require $C^{2}$-linearizability.

We start by noting that Lemmas 4.12 and 4.14 hold without the assumption of $C^{2}$-linearizability. We now distinguish two cases: (a) $\gamma_{N}=\Psi^{N}$, and (b) $\gamma_{N}=N^{v} \Psi^{N}$, $v \geq 1$.

Suppose that case (a) holds. First, we establish upper bounds. By Lemma 4.12, we can reduce to the case when $F$ has only linear constant coefficient terms. Without loss of generality, suppose that $F(x, y)=x_{i}, i \leq S$.

We have

$$
\begin{aligned}
\left|A_{N}\left(x_{i}\right)\right| & =\left|\sum_{n=0}^{N-1}\left(\mu_{i}^{n} A_{i}+C_{i}^{n} \Psi^{N}-\mu_{i}^{n} A_{i}\right)+\sum_{n=N}^{\infty} \mu_{i}^{n} A_{i}\right|, \\
& =\left|\sum_{n=0}^{N-1} C_{i}^{n} \Psi^{N}+\sum_{n=N}^{\infty} \mu_{i}^{n} A_{i}\right|=\tau_{i, N}^{S} \Psi^{N},
\end{aligned}
$$

where

$$
\tau_{i, N}^{s}=\left|\sum_{n=0}^{N-1} C_{i}^{n}+\Psi^{-N} \sum_{n=N}^{\infty} \mu_{i}^{n} A_{i}\right|=\left|\sum_{n=0}^{N-1} C_{i}^{n}\right|+O(1)
$$

Similarly,

$$
\left|A_{N}\left(y_{j}\right)\right|=\tau_{j, N}^{u} \Psi^{N}, \quad 1 \leq j \leq T
$$

Since the constants $C_{i}^{n}$ are uniformly bounded, the $\tau_{\ell, N}^{s / u}$ grow at most linearly in $N$. 
If the sequences $\left(\tau_{\ell, N}^{s / u}\right)$ are all bounded, then the estimates of Lemma 4.13 apply and we proceed as before. Otherwise, choose an unbounded sequence, say $\left(\tau_{i, N}^{s}\right)$, which dominates the other $\left(\tau_{N}\right)$ values (that is, $\tau_{i, N}^{s}=\max \left\{\tau_{\ell, N}^{w} \mid w \in\{u, s\}, \ell\right\}$ ) for infinitely many values of $N$, and along this subsequence $\tau_{i, N}^{s} \rightarrow \infty$. In order to prove Lemma 4.16, we start with a cocycle $F$ based on the function $x_{i}$ (instead of the one provided by Lemma 4.14), and conclude that the inequality (4.12) again holds for infinitely many values of $N$.

Next, we consider case (b), again under the assumption that $\Phi$ is linear on the invariant manifolds through $p$. If we have a $\Psi$-resonance, then Lemma 4.13 holds without the assumption of $C^{2}$-linearizability (the extra factor $N$ that suffices to bound the contribution of the linear part of $F$ is still superseded by $\gamma_{N}$ ). Hence, Lemma 4.14 gives us an optimal lower bound and all goes through as before. If there is no $\Psi$-resonance, then we must introduce the sequences $\left(\tau_{\ell, N}^{s / u}\right)$ as in case (a). In this case, we will have $\left|A_{N}\left(x_{i}\right)\right|=\tau_{i, N}^{s} \beta_{N}$, $i \leq S,\left|A_{N}\left(y_{j}\right)\right|=\tau_{j, N}^{u} \beta_{N}, j \leq T$. If all the $\left(\tau_{\ell, N}^{s / u}\right)$ sequences are bounded, the estimates of Lemma 4.13 apply. Otherwise, we modify the proof of Lemma 4.16 as discussed for case (a).

Finally, we consider the extension of Proposition 4.8 to the case when $\Phi$ is not linear on the local stable and unstable manifolds of $p$. The arguments we present also apply to the proofs of Lemmas 4.12-4.15.

We continue to assume $\Phi$ is $C^{2}$. The first problem is to identify the dominant eigenvalues for the homoclinic orbit-that is, to define the sets $\mathcal{E}_{\star}^{s / u}$. For $m \in \mathbb{N}$, define $\mathbf{A}^{m}=\Phi^{m}(\mathbf{A}), \mathbf{B}^{m}=\Phi^{-m}(\mathbf{B})$. Noting that a weakest contracting eigenvalue is $\mu_{S}$, we may write

$$
\Phi_{S}(\mathbf{x})_{S}=J_{S} \mathbf{x}_{S}+g_{S}(\mathbf{x}),
$$

where $J_{S}$ is a Jordan block with eigenvalue $\mu_{S}$ and $g_{S}$ is $C^{2}$. Since $\left|\mu_{S}\right|$ is maximal among the contracting eigenvalues there are no resonances of the form $\mu_{S}=\Pi \mu_{i}^{n_{i}}$. It follows from a parametrized version of a theorem of Sternberg [25, §5] that there is a $C^{2}$-change of coordinates on $\mathbb{E}^{s}$ which linearizes (4.14) (for the convenience of the reader, we give details in an appendix at the end of the paper). In the new coordinates we have $\Phi_{s}(\mathbf{x})_{S}=J_{S} \mathbf{x}_{S}$. We may repeat this linearization for all contracting eigenvalues $\mu_{i}$ with $\left|\mu_{i}\right|=\left|\mu_{S}\right|$. Either it is the case that for all of these weakest eigenvalues, say $\mu_{i}$, $S \geq i \geq \ell, \mathbf{A}_{i}=0$, or not. In the first case, we observe that $\mathbf{x}_{S}=0, \ldots, \mathbf{x}_{\ell}=0$ defines a $\Phi_{s}$-invariant linear subspace $\mathbb{V}$ of $\mathbb{E}^{s}$. We now restrict attention to $\Phi_{S} \mid \mathbb{V}$ and repeat the linearization process. Observe that there are no resonance problems. In this way, we identify a weakest contraction $\tilde{\mu}$ associated to the homoclinic orbit. Associated to $\tilde{\mu}$ will be a $\Phi_{s}$-invariant subspace $\mathbb{V}^{s}$ of $\mathbb{E}^{s}$. Components of $\Phi_{s} \mid \mathbb{V}^{s}$ associated to $\mu_{i}$ with $\left|\mu_{i}\right|=|\mu|$ will be linearizable. The same process works for $\Phi_{u} \mid \mathbb{E}^{u}$. This gives us sufficient information to define the rates $\beta_{N}, \gamma_{N}$. The proof of Proposition 4.8 now goes through much as before-the fact that components of $\Phi_{s}\left|\mathbb{V}^{s}, \Phi_{u}\right| \mathbb{V}^{u}$ associated to the dominant eigenvalues are linear allows us to push through all of the estimates without difficulty. Although we do not spell out all the details, this representation of $\Phi$ suffices to establish the upper and lower bounds proved previously. The basic idea is that components associated to eigenvalues stronger than the dominant eigenvalues can be dominated by linear maps (weaken eigenvalues slightly). 


\section{A third proof of the Parry and Pollicott theorem}

In $\S 3$, we gave a new proof of the theorem of Parry and Pollicott [23] on the genericity of $C^{\alpha}$-stability of Hölder cocycles. This proof did not require transfer operator techniques. We now briefly sketch a further proof that avoids any direct consideration of symbolic dynamics.

We continue with the notational conventions of $\S 3.2$. Suppose that $X \subset\{1, \ldots, n\}^{\mathbb{Z}}$ is an (abstract) transitive subshift of finite type. Given $\theta \in(0,1)$, let $d(x, y)$ denote the associated metric on $X$ and $F_{\theta}(X)$ be the Banach space consisting of all Lipschitz functions $f: X \rightarrow \mathbb{R}$ for this metric. It follows from a theorem of Williams [26], that $X$ can be represented as a hyperbolic basic set $\tilde{X}$ in the spectral decomposition of a structurally stable diffeomorphism $F$ of $S^{3}$. Indeed, we can require that the $\Omega$-set of $F$ will consist of two hyperbolic fixed points, one attracting and one repelling, $\tilde{X}$, and a further hyperbolic basic set $\tilde{Y}$, where $\tilde{Y}$ is conjugate to the subshift of finite type 'dual' to $X$. Williams's construction is quite elementary and all but the final step (adding a repelling hyperbolic fixed point at $\infty$ ), is carried out in $\mathbb{R}^{3}$. In particular, we may construct $F$ so that there is a cover of $\tilde{X} \subset S^{3}$ by $n$ open neighborhoods $U_{i}$ such that the restriction of $F \mid U_{i}$ is affine linear, say $F \mid U_{i}=A_{i} x+b_{i}$. Further, if we choose $\mu \in(0,1)$ sufficiently small, we may require that each linear map $A_{i}$ is semisimple with a double contracting eigenvalue $\mu$ and a single expanding eigenvalue $\mu^{-1}$. We choose a Riemannian metric on $S^{3}$ which coincides with the usual Euclidean metric on $\mathbb{R}^{3}$ on a neighborhood of $\tilde{X}$. In this situation, the natural conjugacy between $\tilde{X}$ and $X$ is a local isometry if we choose $\theta=\mu$. Moreover, Hölder cocycles of class $C^{\alpha}$ on $\tilde{X}$ ( $C^{\alpha}$ with respect to the Riemannian metric on $S^{3}$ ), correspond exactly to cocycles in $F_{\theta^{\alpha}}(X)$. Hence, we can apply Theorem 2.10 in the case $\alpha \in(0,1]$, to deduce the $F_{\theta^{\alpha}}$ genericity of stable cocycles for all $\alpha \in(0,1]$. Since this result holds for all sufficiently small $\alpha$, we obtain the genericity of stability for all $\theta \in(0,1)$.

\section{Transitivity and ergodicity of compact group extensions}

In this section, we prove Theorems 1.3 and 1.5.

Suppose that $X \subset M$ is a hyperbolic basic set for the $C^{1}$-diffeomorphism $\Phi: M \rightarrow M$. Let $\mu$ be an equilibrium state on $X$. Let $G$ be a compact connected Lie group with Haar measure $h$ and define the product measure $\mu \times h$ on $X \times G$. Let $f \in C^{r}(M, G), r>0$. By Keynes and Newton [16] and Livšic regularity, a necessary and sufficient condition for the non-ergodicity of the skew extension $\Phi_{f}: X \times G \rightarrow X \times G$ is that there exists a nontrivial irreducible unitary representation $R$ of $G$ on $\mathbb{C}^{d}$ for some $d \geq 1$ and a continuous function $w: X \rightarrow S^{2 d-1}$ such that

$$
R(f)^{-1} w=w \circ \Phi .
$$

Here, $S^{2 d-1}$ denotes the unit sphere in $\mathbb{C}^{d}$.

Proposition 6.1. Let $X \subset M$ be a hyperbolic basic set and $\mu$ be an equilibrium state on $X$. If $f \in C^{r}(M, G), r>0$, then:

(1) $\Phi_{f}: X \times G \rightarrow X \times G$ is ergodic if and only if $\Phi_{f}$ is transitive;

(2) if $Z \subset X$ is a locally maximal subshift of finite type and $\Phi_{f} \mid Z \times G$ is transitive, then $\Phi_{f}: X \times G \rightarrow X \times G$ is ergodic. 
Proof. (1) If $\Phi_{f}$ is not ergodic, then (6.15) has a continuous solution, $w: X \rightarrow S^{2 d-1}$. The map $W: X \times G \rightarrow S^{2 d-1}$ defined by $W(x, g)=R(g) w(x)$ is then a non-constant $\Phi_{f}$-invariant continuous function on $X \times G$. Hence, $\Phi_{f}$ cannot be transitive. The converse is trivial.

(2) Assume by contradiction that $\Phi_{f}: X \times G \rightarrow X \times G$ is not ergodic. Then (6.15) has a continuous solution $w: X \rightarrow S^{2 d-1}$. Restricting this solution to $Z$, we conclude, as in (1), that $\Phi_{f} \mid Z \times G$ is not transitive, a contradiction.

PROPOSITION 6.2. Let $X \subset M$ be a locally maximal subshift of finite type for the $C^{2}$-diffeomorphism $\Phi$. Let $\mu$ be an equilibrium state on $X$.

Let $r>0$. Then there exists a $C^{r}$-open and-dense subset $\mathcal{W}_{r}$ of the set of $C^{r}$-cocycles $C^{r}(X, G)$ such that $\Phi_{f}: X \times G \rightarrow X \times G$ is ergodic for all $f \in \mathcal{W}_{r}$. Moreover, if $r \geq 2$, then $\mathcal{W}_{r}$ is $C^{2}$-open in $C^{r}(X, G)$.

Proof. By Field and Parry [14] it suffices to consider the case of toral extensions $G=\mathbb{K}^{m}$. The result follows from Theorem 2.10, Lemmas 2.8 and 2.11, and the discussion of $\$ 2.3$.

THEOREM 6.3. Let $X \subset M$ be a hyperbolic basic set for the $C^{2}$-diffeomorphism $\Phi$. Suppose that $\mu$ is an equilibrium state on $X$.

Let $r>0$. Then there exists a $C^{r}$-open and-dense subset $\mathcal{W}_{r}$ of $C^{r}(X, G)$ such that $\Phi_{f}: X \times G \rightarrow X \times G$ is ergodic for all $f \in \mathcal{W}_{r}$. Moreover, if $r \geq 2$, then $\mathcal{W}_{r}$ is $C^{2}$-open in $C^{r}(X, G)$.

Proof. Transverse homoclinic points are dense in $X$, so by Smale's Theorem 2.1, we can find an $H$-set $Z \subset X$. It follows from Proposition 6.2 that there exists a $C^{r}$-open and -dense subset $\mathcal{W}_{r}$ of $C^{r}(X, G)$ such that $\Phi_{f} \mid Z \times G$ is ergodic for all $f \in \mathcal{W}_{r}$. By Proposition 6.1, $\Phi_{f}: X \times G \rightarrow X \times G$ is ergodic for all $f \in \mathcal{W}_{r}$. The fact that $\mathcal{W}_{r}$ is $C^{2}$-open in $C^{r}(X, G)$, $r \geq 2$, follows from Proposition 6.2.

Remark 6.4. The proof of Theorem 6.3 does not rely heavily on the fact that $X$ is a hyperbolic basic set. In fact, the only properties of $X$ that we require are:

(a) $X$ is a locally maximal invariant set with ergodic measure $\mu$;

(b) $X$ contains a transverse homoclinic orbit for a hyperbolic periodic point; and

(c) Livšic regularity theorems hold on $X$. Specifically, given a compact connected Lie group $G$, we require that for each irreducible unitary representation $R$ of $G$, every measurable solution $w$ to the equation $R(f)^{-1} w=w \circ \Phi$ has a continuous version.

In our proof of Theorem 6.3, we made use of the theorem of Keynes and Newton. In some situations, we have to use a different version of this theorem-for example, in the analysis of partially hyperbolic basic sets [12] where we cannot use the strategy of [14] to reduce to the case where $G$ is either Abelian or semisimple. It is then sometimes worthwhile using a slightly different argument based on maximal transitivity components [8]. We conclude the section by briefly summarizing the main ideas (for more details, presented in the context of partially hyperbolic dynamics, see [12]).

We continue to assume that $X$ is a hyperbolic basic set with equilibrium state $\mu$. Let $f \in C^{r}(X, G)$. It follows from Keynes and Newton [16] that the skew extension $\Phi_{f}: X \times G \rightarrow X \times G$ is $\mu \times h$ ergodic if and only if for every non-trivial irreducible unitary 
representation $\rho: G \rightarrow \mathrm{U}(d)$ of $G$, every $\mu \times h$-measurable $\Phi_{f}$-invariant and $G$-equivariant map $w: X \times G \rightarrow \mathbb{C}^{d}$ is zero (almost everywhere). It follows by Livšic regularity [12, §7], that every measurable $\Phi_{f}$-invariant $G$-equivariant map $w: X \times G \rightarrow \mathbb{C}^{d}$ has a continuous realization. It follows in the usual way that $\Phi_{f}$ is ergodic if and only if $\Phi_{f}$ is transitive.

Let $x \in X$ have dense $\Phi$-orbit in $X$. For any $g \in G$, the closure $T(x, g)$ of the $\Phi_{f}$-orbit through $(x, g)$ projects onto all of $X$. We say $T(x, g)$ is a maximal transitivity component for $\Phi_{f}$ if whenever $T\left(x^{\prime}, g^{\prime}\right) \cap T(x, g) \neq \emptyset$, then $T\left(x^{\prime}, g^{\prime}\right) \subset T(x, g)$. If $T(x, g)$ is a maximal transitivity component, it is easy to see that $\mathcal{P}=\{T(x, g) \mid g \in G\}$ defines a partition of $X \times G$ and that $G$ acts on $\mathcal{P}(h T(x, g)=T(x, h g), h \in G)$. The general question of when maximal transitivity components exist is difficult. (Zorn's lemma implies that every transitive set is contained in a maximal transitive set. However, maximal transitive sets may not be disjoint.) However, transitivity components do exist for skew and principal extensions over hyperbolic basic sets (Brin [8]) and also for certain classes of partially hyperbolic basic sets $[\mathbf{1 2}, \S 7]$.

We have the following useful sufficient condition for transitivity.

Lemma 6.5. Suppose that $\mathcal{P}=\{T(x, g) \mid g \in G\}$ is a partition of $X \times G$ into maximal transitivity components. Then $\Phi_{f}$ will be transitive if we can find a $\Phi$-invariant closed subset $Z$ of $X$ such that $\Phi_{f} \mid Z \times G$ is transitive.

Proof. Suppose $\Phi_{f} \mid Z \times G$ is transitive. It follows that there exists $(z, \gamma) \in Z \times G$ such that the $\Phi_{f}$-orbit of $(z, \gamma)$ is dense in $Z \times G$. For some $h \in G,(z, h) \in T(x, g)$. Hence, $T(x, g) \supset \overline{O(z, h)}=Z \times G$. Therefore, $\gamma T(x, g) \cap T(x, g) \neq \emptyset$, all $\gamma \in G$ and so $T(x, g)=X \times G$.

We may now use Lemma 6.5 and Proposition 6.2 to prove genericity of stable ergodicity.

\section{Transitivity of $\mathbb{R}^{m}$-extensions}

In this section, we prove Theorem 1.7.

Suppose that $\Phi: X \rightarrow X$ is a transitive Anosov diffeomorphism. Niţică and Pollicott [19] showed that under a cohomological condition (valid for all known cases) there exist $C^{\infty}$-cocycles $f: X \rightarrow \mathbb{R}^{m}$ such that $\Phi_{f}: X \times \mathbb{R}^{m} \rightarrow X \times \mathbb{R}^{m}$ is $C^{0}$-stably transitive (that is, $\Phi_{f^{\prime}}$ is transitive for all $f^{\prime}$ sufficiently $C^{0}$ close to $f$ ). Previously, in [18], it was proved that Euclidean group extensions $X \times \mathrm{SE}(m)$ are transitive for a $C^{r}$-open and -dense set of $C^{r}$-cocycles $f: X \rightarrow \operatorname{SE}(m)$ provided $m \geq 4$ is even. The result of [18] holds for any hyperbolic basic set $X$. Density of transitive extensions clearly fails for the group $\mathbb{R}^{m}$.

In this section, we show how to extend the result of Niţică and Pollicott [19] to $\mathbb{R}^{m}$-extensions of arbitrary hyperbolic basic sets. We recover the full strength of their result for hyperbolic attractors. In general, we obtain $C^{2}$-stable transitivity.

First, we recall some definitions from [19]. Let $\Phi: X \rightarrow X$ be a hyperbolic basic set and $f: X \rightarrow \mathbb{R}^{m}$ a Hölder cocycle. Define the skew extension $\Phi_{f}: X \times \mathbb{R}^{m} \rightarrow X \times \mathbb{R}^{m}$. Write $f_{n}=\sum_{j=0}^{n-1} f \circ \Phi^{j}$ and define the periodic data $\mathcal{L}_{f} \subset \mathbb{R}^{m}$ to be $\mathcal{L}_{f}=\left\{f_{n}(x) \mid\right.$ $\left.\Phi^{n} x=x\right\}$. Let $\mathcal{G}_{f}$ be the subgroup of $\mathbb{R}^{m}$ generated by $\mathcal{L}_{f}$. The cocycle $f$ satisfies the separating condition if $\mathcal{L}_{f}$ is not contained on one side of a hyperplane through 0 in $\mathbb{R}^{n}$. 
The separating condition guarantees that the closure of $\mathcal{G}_{f}$ coincides with the closure of the subsemigroup generated by $\mathcal{L}_{f}$. Let $\mathcal{S}^{r}$ denote the set of $C^{r}$-cocycles $f \in C^{r}\left(X, \mathbb{R}^{m}\right)$ that satisfy the separating condition. Note that $\mathcal{S}^{r}$ is an open and non-empty (but not dense) subset of $C^{r}\left(X, \mathbb{R}^{m}\right)$. Moreover, it follows from Bousch [4] that $\Phi_{f}$ is not transitive unless $f \in \mathcal{S}^{r}$.

We can now state the main result of this section.

THEOREM 7.1. Let $X \subset M$ be a hyperbolic basic set for the $C^{2}$-diffeomorphism $\Phi$. Let $r>0$. Then there exists a $C^{r}$-open and-dense subset $\mathcal{W} \subset \mathcal{S}^{r}$ such that $\Phi_{f}: X \times \mathbb{R}^{m} \rightarrow X \times \mathbb{R}^{m}$ is transitive for all $f \in \mathcal{W}$. Moreover, if $r \geq 2$, then $\mathcal{W}$ is $C^{2}$-open in $\mathcal{S}^{r}$. If $X$ is an attractor, then $\mathcal{W}$ is $C^{0}$-open in $\mathcal{S}^{r}$.

We require the following lemma.

LEMMA 7.2. [19] Let $X$ be a hyperbolic basic set and $f \in \mathcal{S}^{r}$ for some $r>0$. Suppose that $\mathcal{G}_{f}$ is dense in $\mathbb{R}^{m}$. Then $\Phi_{f}: X \times \mathbb{R}^{m} \rightarrow X \times \mathbb{R}^{m}$ is transitive.

Given $f=\left(f^{1}, \ldots, f^{m}\right): X \rightarrow \mathbb{R}^{m}$, we define $F=\left(F^{1}, \ldots, F^{m}\right): X \rightarrow \mathbb{K}^{m}$ by setting $F^{j}=\exp \left(2 \pi \imath f^{j}\right)$.

THEOREM 7.3. Let $X$ be a hyperbolic basic set for the $C^{1}$-diffeomorphism $\Phi$. Let $r>0$ and suppose that $f \in \mathcal{S}^{r}$. Then $\Phi_{f}: X \times \mathbb{R}^{m} \rightarrow X \times \mathbb{R}^{m}$ is $C^{r}$-stably transitive if and only if $\Phi_{F}: X \times \mathbb{K}^{m} \rightarrow X \times \mathbb{K}^{m}$ is $C^{r}$-stably transitive.

Proof. It is immediate that transitivity of $\Phi_{f}$ implies transitivity of $\Phi_{F}$. If $\Phi_{F}$ is not stably transitive, then there is an $\tilde{F}, C^{r}$-close to $F$, such that $\Phi_{\tilde{F}}$ is not transitive. Since sufficiently small perturbations are $C^{r}$-homotopic to the identity, $\tilde{F}: X \rightarrow \mathbb{K}^{m}$ lifts to $\tilde{f}: X \rightarrow \mathbb{R}^{m} C^{r}$-close to $f$ and $\Phi_{f}$ is not transitive so that $\Phi_{f}$ is not stably transitive. Hence, stable transitivity of $\Phi_{f}$ implies stable transitivity of $\Phi_{F}$.

Next, we prove the converse direction. Suppose that $\Phi_{F}$ is stably transitive. It suffices to show that $\Phi_{f}$ is transitive. By Lemma 7.2, it suffices to show that $\mathcal{G}_{f}$ is dense in $\mathbb{R}^{m}$. If not, then $\mathcal{L}_{f}$ lies in a subgroup isomorphic to $\mathbb{R}^{m-1} \times \mathbb{Z}$. Hence, there exists a nonzero $k \in \mathbb{R}^{m}$ such that $\exp (2 \pi \imath k \cdot \ell)=1$ for all $\ell \in \mathcal{L}_{f}$. Choose $A \in \mathrm{GL}(m)$ near the identity so that $k^{\prime}=A^{T} k \in \mathbb{Q}^{m}$. Then, $\tilde{f}=A f$ is $C^{r}$-close to $f$ and has the property that $\exp \left(2 \pi \imath k^{\prime} \cdot \ell\right)=1$ for all $\ell \in \mathcal{L}_{\tilde{f}}$. Define $k^{\prime \prime} \in \mathbb{Z}^{m}$ by clearing denominators in $k^{\prime}$. Then

$$
\exp \left(2 \pi \imath k^{\prime \prime} \cdot f_{n}(x)\right)=1 \quad \text { whenever } \Phi^{n} x=x
$$

Let $\tilde{F}$ be the $\mathbb{K}^{m}$-cocycle corresponding to $\tilde{f}$ and define $g=\left(\tilde{F}^{1}\right)^{k_{1}^{\prime \prime}} \cdots\left(\tilde{F}^{m}\right)^{k_{m}^{\prime \prime}}$ : $X \rightarrow \mathbb{K}$. Since $k^{\prime \prime} \in \mathbb{Z}^{m}$, the map $g$ is well defined. Moreover, (7.16) becomes the statement that if $\Phi^{n} x=x$, then $g(x) g(\Phi x) \cdots g\left(\Phi^{n-1} x\right)=1$. By the Livšic periodic point theorem, $g=G \circ \Phi G^{-1}$ for some $G: X \rightarrow \mathbb{K}$ measurable (even Hölder). That is,

$$
\left(\tilde{F}^{1}\right)^{k_{1}^{\prime \prime}} \ldots\left(\tilde{F}^{m}\right)^{k_{m}^{\prime \prime}} G=G \circ \Phi .
$$

It is immediate from Keynes and Newton [16] that $\Phi_{\tilde{F}}$ is not ergodic, and hence (by Livšic regularity) not transitive. Thus $\Phi_{\tilde{F}}$ is a non-transitive $\mathbb{K}^{m}$-extension $C^{r}$-close to $\Phi_{F}$, which is the desired contradiction. 
Remark 7.4. We have proved the equivalence of stable transitivity for $\mathbb{R}^{m}$ - and $\mathbb{K}^{m}$-extensions. The corresponding equivalence for transitivity is false.

Proof of Theorem 7.1. It follows from Theorem 7.3 that an $\mathbb{R}^{m}$-extension satisfying the separating condition is stably transitive precisely when the corresponding $\mathbb{K}^{m}$-extension is stably transitive. Now apply Theorem 6.3.

\section{Mixing for suspension flows}

In this section, we prove Theorem 1.9.

Let $\Phi: X \rightarrow X$ be a hyperbolic basic set. A $C^{r}$-function $f: X \rightarrow \mathbb{R}$ is called a roof function if $f$ is strictly positive. We let $\mathcal{R}^{r}$ denote the space of $C^{r}$-roof functions.

Given a roof function $f$, we define the suspension $X^{f}=\{(x, u) \in X \times[0, \infty)\} / \sim$ where $(x, f(x)) \sim(\Phi x, 0)$ and define the suspension flow $\Phi_{t}^{f}: X^{f} \rightarrow X^{f}$ by $\Phi_{t}^{f}(x, u)=$ $(x, u+t)$ computed subject to identifications.

We fix an equilibrium state on $X^{f}$. However, since mixing is independent of the equilibrium state chosen and is equivalent to both weak mixing and topological weak mixing (see Remarks 1.11), we shall suppress the measure in what follows and usually just refer to 'mixing'.

We now state the main result of this section.

THEOREM 8.1. Let $X \subset M$ be a hyperbolic basic set for the $C^{2}$-diffeomorphism $\Phi$. Let $r>0$. Then there exists a $C^{r}$-open and-dense subset $\mathcal{W} \subset \mathcal{R}^{r}$ of roof functions such that the suspension flow $\Phi_{t}^{f}: X^{f} \rightarrow X^{f}$ is mixing for all $f \in \mathcal{W}$. Moreover, if $r \geq 2$, then $\mathcal{W}$ is $C^{2}$-open in $\mathcal{R}^{r}$. If $X$ is an attractor, then $\mathcal{W}$ is $C^{0}$-open in $\mathcal{R}^{r}$.

Given the roof function $f: X \rightarrow \mathbb{R}$, we define the $\mathbb{K}$-cocycle $F: X \rightarrow \mathbb{K}$ by setting $F=\exp (2 \pi \imath f)$.

THEOREM 8.2. Let $X$ be a hyperbolic basic set for the $C^{1}$-diffeomorphism $\Phi$. Let $r>0$ and suppose that $f \in \mathcal{R}^{r}$. Then $\Phi_{t}^{f}: X^{f} \rightarrow X^{f}$ is $C^{r}$-stably mixing if and only if the circle extension $\Phi_{F}: X \times \mathbb{K} \rightarrow X \times \mathbb{K}$ is $C^{r}$-stably transitive.

Proof. Recall from $\S 2.3$ that $\Phi_{F}: X \times \mathbb{K} \rightarrow X \times \mathbb{K}$ fails to be transitive if and only if there exists an integer $\ell \neq 0$ and a continuous function $w: X \rightarrow \mathbb{K}$ such that

$$
w \circ \Phi=w \exp (2 \pi \imath \ell f) .
$$

On the other hand, the suspension flow $\Phi_{t}^{f}: X^{f} \rightarrow X^{f}$ fails to be weak mixing if and only if there exists a real number $a \neq 0$ and a continuous function $w: X \rightarrow \mathbb{K}$ such that

$$
w \circ \Phi=w \exp (2 \pi \iota a f),
$$

see [21, Proposition 6.2].

It is immediate that weak mixing for $\Phi_{t}^{f}$ implies transitivity for $\Phi_{F}$. If $\Phi_{F}$ is not stably transitive, then there is an $\tilde{F}, C^{r}$-close to $F$, such that $\Phi_{\tilde{F}}$ is not transitive. Since sufficiently small perturbations are $C^{r}$-homotopic to the identity, $\tilde{F}: X \rightarrow \mathbb{K}^{m}$ lifts to $\tilde{f}: X \rightarrow \mathbb{R}^{m} C^{r}$ close to $f$ and $\Phi_{t}^{\tilde{f}}$ is not weak mixing, so that $\Phi_{t}^{f}$ is not stably weak mixing. Hence, stable weak mixing for $\Phi_{t}^{f}$ implies stable transitivity for $\Phi_{F}$. 
Next, we prove the converse direction. Suppose that $\Phi_{F}$ is stably transitive. It suffices to show that $\Phi_{t}^{f}$ is weak mixing. If not, then there exists $a>0$ and $w$ continuous such that $w \circ \Phi=w \exp (2 \pi \imath a f)$. In particular, $\exp \left(2 \pi \imath a f_{n}(x)\right)=1$ whenever $\Phi^{n}(x)=x$. Choose a rational number $\tilde{a}$ close to $a$ and define $\tilde{f}=(a / \tilde{a}) f$. Then, $\tilde{f}$ is $C^{r}$-close to $f$ and has the property that $\exp \left(2 \pi \imath \tilde{a} \tilde{f}_{n}(x)\right)=1$ whenever $\Phi^{n}(x)=x$. Define $b \in \mathbb{Z}$ to be the numerator of $\tilde{a}$. Then,

$$
\exp \left(2 \pi \imath b \tilde{f}_{n}(x)\right)=1 \quad \text { whenever } \Phi^{n} x=x .
$$

Let $\tilde{F}$ be the $\mathbb{K}$-cocycle corresponding to $\tilde{f}$ and define $g=\tilde{F}^{b}: X \rightarrow \mathbb{K}$. Since $b \in \mathbb{Z}$, the map $g$ is well defined. Moreover, (8.17) becomes the statement that if $\Phi^{n} x=x$, then $g(x) g(\Phi x) \cdots g\left(\Phi^{n-1} x\right)=1$. By the Livšic periodic point theorem, $g=G \circ \Phi G^{-1}$ for some $G: X \rightarrow \mathbb{K}$ measurable and hence continuous. We have constructed $G: X \rightarrow \mathbb{K}$ continuous and $b \neq 0$ such that

$$
\tilde{F}^{b} G=G \circ \Phi \text {. }
$$

It follows that $\Phi_{\tilde{F}}$ is a non-transitive $\mathbb{K}$-extension $C^{r}$-close to $\Phi_{F}$, which is the desired contradiction.

Proof of Theorem 8.1. It follows from Theorem 8.2 that a roof function defines a stably mixing suspension flow precisely when the corresponding $\mathbb{K}$-extension is stably transitive. Now apply Theorem 6.3.

Acknowledgement. Research supported in part by NSF grant DMS-0071735 and EPSRC grant GR/R87543/01.

\section{A. Appendix. Linearization along part of an invariant manifold}

In this appendix we provide a proof of the partial linearization statements made in $\$ 4.9$. Our method is elementary and based on that used by Sternberg in his proof of Poincaré's theorem [25].

Let $U$ be an $n$-dimensional real vector space. Let $k \geq 2$, and suppose that $F: U \rightarrow U$ is a $C^{k}$-diffeomorphism such that $D F(0)$ has all eigenvalues of modulus strictly less than 1 . Denote the set of eigenvalues of $D F(0)$ by $\mathcal{E}$ and set $S=\max \{\mid \mu \| \mu \in \mathcal{E}\}$. We have a $D F(0)$-invariant splitting $U=V \oplus W$, where $D F(0) \mid V=L$ has all eigenvalues of modulus equal to $S$ and all eigenvalues of $D F(0) \mid W=M$ have modulus strictly less than $S$. Denote coordinates on $V \oplus W$ by $(x, p)$.

Proposition A.1. There exists a local $C^{k}$-diffeomorphism $H: V \oplus W \rightarrow V \oplus W$; $(x, p) \mapsto(h(x, p), p)$ which linearizes $F$ along $V$ :

$$
h(F(x, p))=\operatorname{Lh}(x, p), \quad(x, p) \in V \oplus W .
$$

That is, $H \circ F \circ H^{-1}=(L, G)$, where $G: V \oplus W \rightarrow W$ is $C^{k}$ and $D G(0)=M$.

We provide a proof of Proposition A.1 in the simplest case when $V$ is one-dimensional (and so we can write $L x=S x, x \in \mathbb{R}$ ) and $k$ is an integer. The method we present works, with trivial modifications, when there are multiple eigenvalues of modulus equal to $S, L$ is not semisimple and when we allow $k$ to be non-integral. 
Henceforth, we write $U=\mathbb{R} \oplus W$. Choose a Euclidean norm \|\| on $U$ such that $\mathbb{R} \perp W,\|D F(0)\|=S$ and $\|D F(0) \mid W\|=s<S$. Let $B_{R}$ denote the closed $R$-ball, center the origin, in $U$.

For $R>0$, we let $\mathcal{F}_{R}^{k}$ denote the space of $\mathbb{R}$-valued $C^{k}$-functions defined on $B_{R}$ such that $\partial^{\alpha} f(0)=0$ for all $f \in \mathcal{F}_{R}^{k},|\alpha| \leq k$. Define a norm \|\|$_{R, k}$ on $\mathcal{F}_{R}^{k}$ by

$$
\|f\|_{R, k}=\sup _{(x, p) \in B_{R}}\left\|D^{k} f(x, p)\right\|
$$

where we regard $D^{k} f(x, p)$ as a symmetric $k$-linear map and take the corresponding operator norm.

Lemma A.2. (cf. [25, Lemma 2]) There exists $C=C_{k}>0$ such that for $0 \leq j \leq k-1$ and all $f \in \mathcal{F}_{R}^{k}$ we have

$$
\|f\|_{R, j} \leq C R^{k-j}\|f\|_{R, k}
$$

Proof. A simple consequence of Taylor's theorem with integral remainder together with the fact that elements of $\mathcal{F}_{R}^{k}$ are $k$-flat at the origin.

It follows from the lemma that $\left(\mathcal{F}_{R}^{k},\|\|_{R, k}\right)$ is a Banach space.

Following Sternberg [25], we define the operator $\mathcal{D}_{F}: \mathcal{F}_{R}^{k} \rightarrow \mathcal{F}_{R}^{k}$ by

$$
\mathcal{D}_{F}(h)(x, p)=L^{-1} h(F(x, p)), \quad h \in \mathcal{F}_{R}^{k} .
$$

Since $D F(0)$ is a contraction, it follows that, provided $R$ is sufficiently small, $F\left(B_{R}\right) \subset$ $B_{R}$ and so $\mathcal{D}_{F}$ is well defined.

LEMmA A.3. (cf. [25, Lemma 3]) We can choose $R>0$ and $K \in(0,1)$ such that

$$
\left\|\mathcal{D}_{F}(h)\right\|_{R, k} \leq K\|h\|_{R, k}, \quad h \in \mathcal{F}_{R}^{k} .
$$

Proof. This estimate is a parametrized version of part of [25, Lemma 3]. Since $k \geq 2$, we may choose $\delta>0$ so that $(S+\delta)^{k} / S<1$. Now, fix $\varepsilon>0$ so that $K=$ $\left[(S+\delta)^{k}+\varepsilon\right] / S<1$. Let $h \in \mathcal{F}_{R}^{k}$. We have

$$
D^{k}(h \circ F)=\left(D^{k} h\right) F(D F)^{k}+P_{\alpha},
$$

where $P_{\alpha}$ involves derivatives of order less than $k$ of $h$. Since $S$ is the weakest contracting eigenvalue, we can choose $R>0$ sufficiently small so that $\|D F\|$ is bounded by $S+\delta$ on $B_{R}$. Hence, $\left\|\left(D^{k} h\right) F(D F)^{k}\right\| \leq(S+\delta)^{k}\left\|\left(D^{k} h\right) F\right\|$ on $B_{R}$. It follows from Lemma A.2 that we can make $\left\|P_{\alpha}\right\|_{R, 0}$ an arbitrarily small multiple of $\|h\|_{R, k}$ by taking $R$ small enough. Hence, just as in [25, Lemma 3], we may choose $R>0$ so that

$$
\left\|\mathcal{D}_{F}(h)\right\|_{R, k} \leq S^{-1}\left[(S+\delta)^{k}+\varepsilon\right]\|h\|_{R, k}, \quad h \in \mathcal{F}_{R}^{k},
$$

where $\varepsilon, \delta>0$ were chosen above.

Since there can be no resonances relating a weakest eigenvalue of $D F(0)$ to products of other contracting eigenvalues-in our case, $S \neq \Pi \mu_{i}^{m_{i}}$-we can make a local $C^{k}$-change of coordinates $H_{0}(x, p)=\left(h_{0}(x, p), p\right)$ on $U$ such that $h_{0} \circ F-L h_{0} \in \mathcal{F}_{R}^{k}$. Composing on the left by $L^{-1}$, we have

$$
h_{0}-L^{-1} h_{0} \circ F \in \mathcal{F}_{R}^{k}
$$


Applying Lemma A.3, we see that the sequence

$$
h_{n}=L^{-n} h_{0} F^{n}=\sum_{j=0}^{n-1} \mathcal{D}_{F}^{j}\left(L^{-1} h_{0} \circ F-h_{0}\right)+h_{0},
$$

is uniformly convergent to a $C^{k}$-function $h: B_{R} \rightarrow \mathbb{R}$. Obviously, $h$ satisfies the conditions of Proposition A.1.

\section{REFERENCES}

[1] R. Adler, B. Kitchens and M. Shub. Stably ergodic skew products. Discrete Contin. Dyn. Syst. 2 (1996), 349-350 (Errata Discrete Contin. Dyn. Syst. 5 (1999), 456.)

[2] D. V. Anosov. Geodesic flows on closed Riemannian manifolds with negative curvature. Proc. Steklov Inst. Math. 90 (1967).

[3] C. Bonatti, X. Gomez-Mont and M. Viana. Généricité d'exposants de Lyapunov non-nuls pour des produits déterministes de matrices. Ann. Inst. H. Poincaré C 20(4) (2003), 579-624.

[4] T. Bousch. La condition de Walters. Ann. Sci. École Norm. Sup. (4) 34 (2001), 287-311.

[5] R. Bowen. Periodic points and measures for Axiom A diffeomorphisms. Trans. Amer. Math. Soc. 154 (1971), 377-397.

[6] R. Bowen. Equilibrium States and the Ergodic Theory of Axiom A Diffeomorphisms (Springer Lecture Notes in Mathematics, 470). Springer, Berlin, 1975.

[7] R. Bowen and D. Ruelle. The ergodic theory of Axiom A flows. Invent. Math. 29 (1975), 181-202.

[8] M. I. Brin. Topology of group extensions of Anosov systems. Mathematical Notes of the Acad. of Sciences of the USSR 18(3) (1975), 858-864.

[9] M. I. Brin. Topological transitivity of a class of dynamical systems and frame flows on manifolds of negative curvature. Funktsional. Anal. i Prilozhen. 9(1) (1975), 9-19.

[10] M. I. Brin and J. B. Pesin. Partially hyperbolic dynamical systems. Math. USSR Izvestia 8 (1974), 177-218.

[11] M. J. Field, I. Melbourne and A. Török. Stability of mixing and rapid mixing for hyperbolic flows. Preprint, 2004.

[12] M. J. Field and M. Nicol. Ergodic theory of equivariant diffeomorphisms: Markov partitions and stable ergodicity. Mem. Amer. Math. Soc. 803 (2004).

[13] M. J. Field and V. Niţică. Stable topological transitivity of skew and principal extensions. Nonlinearity 14 (2001), 1055-1070.

[14] M. J. Field and W. Parry. Stable ergodicity of skew extensions by compact Lie groups. Topology, 38(1) (1999), 167-187.

[15] A. Katok and B. Hasselblatt. Introduction to the Modern Theory of Dynamical Systems (Encyclopedia of Mathematics and its Applications, 54). Cambridge University Press, Cambridge, 1995.

[16] H. B. Keynes and D. Newton. Ergodic measures for nonabelian compact group extensions. Compositio Math. 32 (1976), 53-70.

[17] A. N. Livšic. Cohomology of dynamical systems. Math. USSR Izvestija 6(6) (1972), 1278-1301.

[18] I. Melbourne and M. Nicol. Stable transitivity of Euclidean group extensions. Ergod. Th. \& Dynam. Sys. 23 (2003), 611-619.

[19] V. Niţică and M. Pollicott. Transitivity of Euclidean extensions of Anosov diffeomorphisms. Ergod. Th. \& Dynam. Sys. (2003), to appear.

[20] W. Parry. 'Skew-products of shifts with a compact Lie group', J. London Math. Soc.(2) 56 (1997), 400-404.

[21] W. Parry and M. Pollicott. Zeta functions and the periodic orbit structure of hyperbolic dynamics. Astérisque 187-188 (1990).

[22] W. Parry and M. Pollicott. The Livsic cocycle equation for compact Lie group extensions of hyperbolic systems. J. London Math. Soc. (2) 56 (1997), 405-416.

[23] W. Parry and M. Pollicott. Stability of mixing for toral extensions of hyperbolic systems. Proc. Steklov Inst. Math. 216 (1997), 354-363.

[24] M. Pollicott. On the rate of mixing of Axiom A flows. Invent. Math. 81 (1985), 427-447.

[25] S. Sternberg. Local contractions and a theorem of Poincaré'. Amer. J. Math. 79 (1957), 809-824.

[26] R. F. Williams. Classification of sub-shifts of finite type. Ann. Math. 68 (1973), 120-153. 